\title{
Plant development regulated by cytokinin sinks
}

Zurcher, E ; Liu, J ; di Donato, M ; Geisler, M ; Muller, B

DOI: https://doi.org/10.1126/science.aaf7254

Posted at the Zurich Open Repository and Archive, University of Zurich ZORA URL: https://doi.org/10.5167/uzh-131624

Journal Article

Accepted Version

Originally published at:

Zurcher, E; Liu, J; di Donato, M; Geisler, M; Muller, B (2016). Plant development regulated by cytokinin sinks. Science, 353(6303):1027-1030.

DOI: https://doi.org/10.1126/science.aaf7254 


\title{
Title: Plant development regulated by cytokinin sinks
}

\author{
Authors: Evelyne Zürcher ${ }^{1} \dagger$, Jingchun $\operatorname{Liu}^{1} \dagger$, Martin di Donato ${ }^{2}$, Markus Geisler ${ }^{2} \&$ Bruno \\ Müller ${ }^{1 *}$
}

\begin{abstract}
Affiliations:
${ }^{1}$ University of Zurich, Department of Plant and Microbial Biology, Zurich-Basel Plant Science Center, 8008 Zurich, Switzerland.

${ }^{2}$ University of Fribourg, Department of Biology, Plant Biology, 1700 Fribourg, Switzerland.

*Correspondence to: bruno.mueller@uzh.ch.

$\dagger$ These authors contributed equally.
\end{abstract}

\begin{abstract}
Morphogenetic signals control patterning of multicellular organisms. Cytokinins are mobile signals that are perceived by subsets of plant cells. Here, we show that the responses to cytokinin signaling during Arabidopsis development are constrained by the transporter PURINE PERMEASE 14 (PUP14). PUP14 is inversely expressed to the cytokinin signaling readout. The loss of PUP14 function allows ectopic cytokinin signaling accompanied by aberrant morphogenesis in embryos, roots and the shoot apical meristem. PUP14 protein localizes to the plasma membrane and imports bioactive cytokinins, thus depletes apoplastic cytokinin pools and inhibits perception by plasma-membrane localized cytokinin sensors. We propose that the spatiotemporal cytokinin sink patterns established by PUP14 determine the cytokinin signaling landscape shaping morphogenesis of land plants.
\end{abstract}

One Sentence Summary: Cytokinin import mediated by Arabidopsis PURINE PERMEASE 14 confines the cytokinin signaling-patterning landscape to control morphogenesis.

Main Text: Multicellular organisms depend on differential cell functions controlled by signaling systems. The precise determination of signal-perceiving cells is important to ensure normal development. Cytokinins are chemical plant signals that control morphogenesis, integrate environmental cues, and mediate biotic interactions (1-3). Cytokinins are perceived by largely redundantly acting hybrid kinases that activate a phosphorelay circuitry to stimulate transcription of target genes. The spatiotemporal precision of the signaling patterns in the different plant organs $(4,5)$ raises the question of how control is established. Each step involved in eliciting a signaling response, including ligand biosynthesis or expression of signaling components, may be differentially regulated and contribute to defining the signaling patterns. To identify limiting and regulated steps, we used Arabidopsis heart-stage embryos as a model where the cytokinin 
response marks the provascular tissue (Fig. 1A). First, to evaluate whether bioactive cytokinins are limited, embryos were incubated for $16 \mathrm{~h}$ with the degradation-insensitive cytokinin benzyl adenine (BA) (6). This caused a stereotypic expansion of the synthetic cytokinin reporter TCSn::GFP (Two Component signaling Sensor new:: green fluorescent protein) (5) (Fig. 1A), confirming that cytokinin levels are controlled (7). However, excess cytokinins did not induce TCSn::GFP expression in the prospective cotyledons. This is despite the transcription of the cognate cytokinin receptor ARABIDOPSIS HISTIDINE KINASE 4 (AHK4) in these domains (Fig. 1A), suggesting that failure to turn on signaling cannot be explained by missing receptors. To test whether signaling downstream of receptors is functional, we expressed CYTOKININ INDEPENDENT 1 (CKI1). CKI1 encodes a hybrid kinase with cytokinin-independent constitutive activity (3). Its short-term expression caused ubiquitous TCSn::GFP activation (Fig. 1A). Together, these results suggest that cells of the prospective cotyledons fail to activate cytokinin signaling despite a functional signaling system, and even upon addition of abundant active ligand. We hypothesized that productive ligand-receptor interactions within organs could depend on cytokinin transporters that guide differential cellular localization of cytokinins. To test whether members of the Arabidopsis PURINE PERMEASES (PUPs) family of transmembrane proteins implicated in cytokinin translocation (8) control the spatio-temporal landscape of cytokinin signaling, we first established a transcription profile of all family members based on our own analysis and published transcriptome data (9-11) (fig. S1). PUP14 expression was unique to prevail in all organs and stages analyzed, including embryos. To determine the PUP14 expression pattern, we analyzed PUP 14::PUP 14-GFP transgenic plants. In heart-stage embryos, PUP14-GFP localized to cells that failed to respond to cytokinins including cells of the prospective cotyledons (Fig. 1A). We confirmed this pattern by mRNA in situ hybridization with a PUP14 antisense probe (Fig. 1B, fig. S2A). The exclusive nature of PUP14 expression and the cytokinin signaling pattern is compatible with an inhibitory function of PUP14 in the cytokinin response. To eliminate PUP14 function during defined time windows, which avoids secondary effects and potential lethality issues, we constructed an ethanol-inducible artificial microRNA (amiR) (12) targeting PUP14 (35S > ALC >amiRPUP14). Upon induction of the amiRPUP14 transgene, PUP14 mRNA and PUP14-GFP levels were reduced within $24 \mathrm{~h}$ (Fig. 1D, fig. S2, B and C). The amiRPUP14-induced phenotypes were complemented by an amiRPUP14-resistant transgene (PUP14*) encompassing the PUP14 locus (fig. S2, E to G), suggesting that the inducible amiRPUP14 acts specifically. In addition, an inducible $a m i R$ against non-expressed PUP19 and PUP20 (fig. S1) did not cause obvious phenotypes (fig. S2, E to G). Finally, a TDNA insertion to the PUP14 promoter causing a reduction in PUP14 mRNA levels showed qualitatively similar but weaker phenotypes in embryos, seedlings and adult shoots compared to amiRPUP14-induced phenotypes (Fig. 1C, fig. S3, A to F), while a second T-DNA insertion 3prime of the PUP14 locus did not affect PUP14 mRNA levels (fig. S3B) and produced no apparent phenotypes. Together, these results validate the use of the amiRPUP14 line to study PUP14 function. Inducing amiRPUP14 expression for $16 \mathrm{~h}$ caused widespread ectopic cytokinin signaling in the embryo (Fig. 1B), also in cells of the prospective cotyledons that are nonresponsive to treatments with exogenous cytokinins (Fig. 1A), supporting the role of PUP14 in confining the cytokinin response. The same treatment regime did not affect the auxin response (fig. S2D), indicating that PUP14 acts specifically on cytokinin signaling. After $2 \mathrm{~d}$ of amiRPUP14 induction, morphological defects in the prospective cotyledons and the nascent root meristem became apparent (Fig. 1B), consistent with the ectopic cytokinin responses in these domains. As loss of PUP14 produces ectopic cytokinin responses, we expected the 
overexpression of PUP14 to reduce cytokinin output. While we were unable to recover plants transgenic for $35 S:: P U P 14$, inducible PUP14 expression in the embryo reduced the endogenous cytokinin response after $16 \mathrm{~h}$, and after $48 \mathrm{~h}$ of transgene induction, morphological defects in the embryo root were apparent (Fig. 1C). Similar to the embryo, we found PUP14 expression in the meristematic region of the seedling's main root (fig. S4A), the lateral root primordia (LRP) (fig. S4D), and in ovules and seeds (fig. S5, A and B) exhibiting complementary patterns to those of cytokinin signaling. As in the embryo, short-term amiRPUP14 induction resulted in ectopic cytokinin signaling in the seedling root, particular in the meristematic region of the root tip (fig. $\mathrm{S} 4 \mathrm{~A}$ ) and in the LRP (fig. S4B). Accordingly, transcription of the immediate-early cytokinin target genes type-A ARABIDOPSIS RESPONSE REGULATORS (ARR) ARR5, 6 and 7 (13) was induced in seedlings (Fig. 1C). Continuous induction of amiRPUP14 led to growth retardation of the seedling root and shoot, and a suppression of lateral roots $(14,15)$ (Fig. 1E), suggesting that the root and shoot meristem activities were both affected when cytokinin signaling patterns were perturbed by the inducible amiRPUP14. In contrast to the embryo (Fig. 1B), TCSn::GFP expression remained unchanged after $24 \mathrm{~h}$ of inducing ectopic PUP14 in the seedling root (not shown). We visualized PUP14-GFP expressed from the inducible $35 S>A L C>P U P 14-G F P$ transgene and found that, compared to $35 S>A L C>G F P$, cells of the root apex and vasculature failed to express PUP14-GFP (fig. S5, A to C) suggesting that ectopic PUP14 is not tolerated, which can explain the absent effects on TCSn::GFP in the seedling root apex, and the lethality of the $35 S:: P U P 14$ transgene. In addition, the subcellular localization of ectopic PUP14-GFP was disturbed compared to endogenous PUP14-GFP (fig. S5, B to C), which may impair its normal function. In the shoot, cytokinin controls the homeostasis of the shoot apical meristem (SAM) (16), where increased cytokinin causes a more active meristem with more primordia (17). As observed in other developmental contexts (Fig. 1, A to B, figs. S4, S6, A and B), PUP14 expression in the SAM was inversely correlated with cytokinin signaling output assayed by TCSn::GFP (Fig. 2A). Inducing amiRPUP14 expression in adult plants that were allowed to complete embryogenesis and the early vegetative phase of development undisturbed caused ectopic cytokinin output in the SAM, which was accompanied by a $37 \%$ more primordia, $94 \%$ increased shoot branching and disturbed phyllotaxis (Fig. 2, B and C). Similar phenotypes have been observed in plants mutant for CYTOKININ OXIDASE (CKX) 3 and 5 (17), ARABIDOPSIS HISTIDINE PHOSPHOTRANSFER PROTEIN 6 (18), and ARR3-9 (19), which also display ectopic cytokinin activities. Thus, PUP14 functions to limit the cytokinin response domains throughout development to support morphogenesis.

Next, we addressed the cellular function of PUP14. PUP14-GFP fusion proteins, supported by BFA-sensitive vesicular transport, localize to the plasma membrane (fig. S6C). To test PUP14's cytokinin transport capacity, we conducted uptake experiments using labelled trans-zeatin (tZ), an abundant natural cytokinin (20). Transient expression of PUP14 in mesophyll protoplasts or tobacco microsomes stimulated the uptake of labelled tZ (Fig. 3, A and B). The PUP14 transport activity was ATP-dependent and higher compared to PUP1 (8) (Fig. 3B). Uptake was inhibited by unlabeled $t Z$, by the common natural cytokinin isopentenyl adenine (iP), by the aromatic cytokinin $\mathrm{BA}$, and also by adenine, but not by $\mathrm{tZ}$ riboside, the major cytokinin transport form (21), nor auxin (IAA), nor allantoin, which is an unrelated substrate (Fig. 3, C and D). Energydependent cytokinin uptake into a microsomal cell-free system excludes that uptake is dependent on cytoplasmic metabolization. Conversely, seedlings with decreased PUP14 levels exhibited a reduced uptake rate for exogenously added $\mathrm{tZ}$ compared to control seedlings (Fig. 3E). Our data show that plasma membrane-localized PUP14 imports bioactive cytokinins to the 
cytosol, implying that PUP14 activity depletes ligands from the apoplast, which leads to a suppression of the cytokinin response. In this scenario, extracellular cytokinins binding to the sensing domains of plasma membrane-localized receptors $(22,23)$ are important to initiate the signaling response, while the cytoplasm represents a sink for bioactive ligands. To test this hypothesis, we devised experiments that compare the effects of differentially targeted cytokinindegrading enzymes on the cytokinin signaling response. Mesophyll protoplast cells responded to as little as $100 \mathrm{pM}$ of exogenously added tZ by activating cytokinin signaling (4), suggesting they depend on exogenous cytokinins, and thus serve as a suitable model to study cytokinin perception independent of production. Transient transfection of PUP14 localizing to the plasma membrane (fig. S7A) caused a reduction of cytokinin-dependent TCS::LUCIFERASE (LUC) activity (Fig. 3F), recapitulating the phenotypes from PUP14 overexpression in the embryo (Fig. 1B). Transient expression of wild-type CKX2 that is targeted for secretion to the apoplast (20) (fig. S7B) attenuated the cytokinin response triggered by $\mathrm{tZ}$ but not by the degradation-resistant BA. To target CKX2 to the exofacial side of the plasma membrane, we added a glycosyl phosphatidyl inositol (GPI)-anchor (24) resulting in CKX2-GPI (fig. S7C), which also caused a reduction in the cytokinin response. In contrast, a variant of CKX2 that lacks the N-terminal signal peptide ( $\triangle$ SP-CKX2) and co-localizes with a cytoplasmic marker (fig. S7D) did not affect the cytokinin response, and neither did CKX7, which also localizes to the cytoplasm (25) (fig. S7E). Crude cell extracts obtained from $\triangle$ SP-CKX2- or CKX7-transfected cells added to the medium reduced the response triggered by $\mathrm{tZ}$, indicating these proteins are active. These data suggest that apoplastic cytokinins initiate signaling, while cytoplasmic cytokinins are inactive.

PUP14 imports cytokinins from the apoplast to the cytosol, away from sensing domains of plasma membrane-localized receptors, which causes a reduction in cytokinin signaling. Thus, PUP14 activity inversely correlates with the capacity of a cell to sense cytokinins (fig. S8), and PUP14 spatio-temporal activities cause region-specific depletion of cytokinins from the apoplast. In animals, the importance of such clearing activities is demonstrated by the powerful action of drugs that target dopamine influx transporters thereby increasing its residence time in the synaptic cleft (26). Feeding experiments with radiolabeled bioactive cytokinin suggested that the bulk of imported cytokinins are inactivated by conversion to monophosphates by ADENINE PHOSPHORIBOSYL TRANSFERASE enzymes $(27,28)$. Furthermore, N- or O-glycosylation, oxidative cleavage, or transport to other cells may contribute to clearance of intracellular cytokinins (2). PUP14 is the only family member to be linked to cytokinin signaling in all organs. In specific developmental contexts, additional PUP family members likely have overlapping functions with PUP14. The fact that $P U P$ genes are specific to vascular plants (29) may suggest $P U P$ genes are needed to support more complex cytokinin signaling patterns associated with the bauplan of land plants. As hormonal transporters are numerous and universal in plant and animal systems, transporters in other systems may also regulate patterning during morphogenesis.

\section{References and Notes:}

1. M. Miri, P. Janakirama, M. Held, L. Ross, K. Szczyglowski, Trends Plant Sci. 21, 178 (2016).

2. J. J. Kieber, G. E. Schaller, Arabidopsis Book 12, e0168 (2014).

3. I. Hwang, J. Sheen, B. Müller, Annu. Rev. Plant Biol. 63, 353 (2012).

4. B. Müller, J. Sheen, Nature 453, 1094 (2008). 
5. E. Zürcher et al., Plant Physiol. 161, 1066 (2013).

6. P. Galuszka et al., J Plant Growth Regul 26, 255 (2007).

7. B. De Rybel et al., Science 345, 1255215 (2014).

8. B. Gillissen et al., Plant Cell 12, 291 (2000).

9. J. Adrian et al., Dev. Cell 33, 107 (2015).

10. M. F. Belmonte et al., Proc. Natl. Acad. Sci. U S A 110, E435 (2013).

11. R. K. Yadav, M. Tavakkoli, M. Xie, T. Girke, G. V. Reddy, Dev. 141, 2735 (2014).

12. R. Schwab, S. Ossowski, M. Riester, N. Warthmann, D. Weigel, Plant Cell 18, 1121 (2006).

13. I. B. D'Agostino, J. Deruère, J. J. Kieber, Plant Physiol. 124, 1706 (2000).

14. L. Laplaze et al., Plant Cell 19, 3889 (2007).

15. J. P. To et al., Plant Cell 16, 658 (2004).

16. S. P. Gordon, V. S. Chickarmane, C. Ohno, E. M. Meyerowitz, Proc. Natl. Acad. Sci. U S A 106, 16529 (2009).

17. I. Bartrina, E. Otto, M. Strnad, T. Werner, T. Schmülling, Plant Cell 23, 69 (2011).

18. F. Besnard et al., Nature 505, 417 (2014).

19. A. Leibfried et al., Nature 438, 1172 (2005).

20. T. Werner et al., Plant Cell 15, 2532 (2003).

21. C. A. Beveridge et al., Plant J. 11, 339 (1997).

22. H. J. Kim et al., Proc. Natl. Acad. Sci. U S A 103, 814 (2006).

23. K. Wulfetange et al., Plant Physiol. 156, 1808 (2011).

24. A. Baral et al., Plant Cell 27, 1297 (2015).

25. I. Köllmer, O. Novák, M. Strnad, T. Schmülling, T. Werner, Plant J. 78, 359 (2014).

26. R. A. Vaughan, J. D. Foster, Trends Pharmacol Sci 34, 489 (2013).

27. B. Moffatt, C. Pethe, M. Laloue, Plant Physiol. 95, 900 (1991).

28. X. Zhang et al., Mol Plant 6, 1661 (2013).

29. S. B. Hildreth et al., Proc. Natl. Acad. Sci. U S A 108, 18179 (2011).

30. D. R. Smyth, J. L. Bowman, E. M. Meyerowitz, Plant Cell 2, 755 (1990).

31. D. S. Lituiev et al., Dev. 140, 4544 (2013).

32. S. J. Clough, A. F. Bent, Plant J. 16, 735 (1998).

33. H. A. Roslan et al., Plant J. 28, 225 (2001).

34. C. Xiang, P. Han, I. Lutziger, K. Wang, D. J. Oliver, Plant Mol. Biol. 40, 711 (1999).

35. C. Aslanidis, P. J. de Jong, Nucleic Acids Res. 18, 6069 (1990).

36. B. De Rybel et al., Plant Physiol. 156, 1292 (2011).

37. S. D. Yoo, Y. H. Cho, J. Sheen, Nat Protoc 2, 1565 (2007).

38. A. Bielach et al., Plant Cell 24, 3967 (2012).

39. S. Henrichs et al., EMBO J. 31, 2965 (2012).

40. K. J. Livak, T. D. Schmittgen, Methods 25, 402 (2001).

41. G. Jach, E. Binot, S. Frings, K. Luxa, J. Schell, Plant J. 28, 483 (2001).

Acknowledgments: We thank Matthias Philipp for technical assistance, Ioanna Antoniadi and Anja Schmidt for sharing unpublished results, Enrico Martinoia (E.M.) for help with transport assays, Jen Sheen and Konrad Basler for critical discussions and comments on the manuscript, Célia Baroux for comments on the manuscript, and Ueli Grossniklaus for 
support. Funding was provided by the Kanton of Zurich, the Swiss National Science Foundation (SNF31003A-149459), and by a Syngenta PhD Fellowship from the ZurichBasel Plant Science Center. The authors declare that they have no conflicts of interest. The supplementary materials contain additional data. Plasmid sequences were deposited to Genbank, accession numbers are KX510271-KX510275.

\section{Fig. 1. PUP14 function in embryo and seedlings.}

(A) Heart-stage embryos subjected to $16 \mathrm{~h}$ mock, $16 \mathrm{~h} 10 \mu \mathrm{M}$ BA treatment, hybridized with $A H K 4$ antisense (as) RNA, and $16 \mathrm{~h} C K I 1$ expression from a $35 S>A L C>C K I 1$ transgene (5) (CKIox). GFP reporter transgenes as indicated. (B) PUP14 expression detected by reporter gene and by PUP14 as RNA probe. amiRPUP14 and PUP14 transgene inductions, ectopic TCSn::GFP in $85 \%$ of embryos, $\mathrm{n}=53$, loss of TCSn::GFP in $45 \%$ of embryos, $\mathrm{n}=11$. (C) Morphological defects $48 \mathrm{~h}$ after transgene inductions and in pup14-1 (for amiRPUP14 47\%, $\mathrm{n}=96$, for pup 14-1, 37\%, $\mathrm{n}=237$, for PUP14 overexpression from $35 S>A L C>P U P 14$ [PUP14ox], $50 \%, \mathrm{n}=10$ ), (arrowheads point to affected cotyledons, arrow to shortened embryo root, cell boundaries in root meristem outlined with white dotted lines). (D) Relative changes of type-A $A R R 5,6$ and 7, and TCSn::GFP (as a group significantly different: $\mathrm{p}<0.001$ from unpaired t-test, TCSn::GFP: $\mathrm{n}=4 ;$ TCSn::GFP, amiRPUP14: $\mathrm{n}=4$ ) and PUP14 mRNA levels (significantly different: $\mathrm{p}<0.001$ from unpaired t-test, TCSn::GFP $: \mathrm{n}=4$; TCSn $: \because G F P$, amiRPUP14: $\mathrm{n}=7$ ) after $16 \mathrm{~h}$ of amiRPUP14 induction in $7 \mathrm{~d}$ old seedlings of indicated genotype, assessed by quantitated real-time (qRT)-PCR, error bars represent s.e.m. (E) Seedlings after $7 \mathrm{~d}$ on ethanol-containing medium. Growth retardation of seedling roots, $n=10$. Scale bars (A to B) $20 \mu \mathrm{m},(\mathrm{E}) 1 \mathrm{~cm}$.

\section{Fig. 2. PUP14 confines the cytokinin response in the SAM.}

(A) Floral SAM. Longitudinal optical sections in lower panels at cyan-colored brackets, dotted lines mark organ boundaries. Transgenes indicated. Arrows indicate peak PUP14-GFP levels at organ-organ boundaries. Ectopic TCSn::GFP (arrowheads) after amiRPUP14 induction. (B-C) Comparisons of ethanol-treated Col0 and amiRPUP14 phenotypes. (B) Inflorescences and inflorescence stems, red dots denote flower primordia, arrowheads indicate perturbations. (C) Numbers of flower primordia, at stages 6-12 on the main apex (30), $n=6$. Number of primary rosette $(\mathrm{RI})$ and primary cauline branching $(\mathrm{CI}), \mathrm{n}=6$. Data represent mean values, error bars represent s.d. $* *$ p $<0.01$ unpaired t-test. Scale bars (A) $20 \mu \mathrm{m}$, (B) $1 \mathrm{~mm}$ (flower primordia), 1 $\mathrm{cm}$ (stems).

\section{Fig. 3. PUP14 cellular function.}

(A-E) PUP14 transport assays, with relative C14-tZ uptake rates on y-axis. (A) PUP14-transfected mesophyll protoplasts. (B) Microsomes derived from GFP, PUP1, or PUP14 transfected N. benthamiana. (C) Competition by indicated substances in PUP14-transfected protoplasts. (D) Competition in microsomes of $35 S:: P U P 14$ transfected $N$. benthamiana. (E) amiRPUP14 vs. Col0 seedlings. (F) Relative TCSn::LUC inductions in protoplasts, treated by $10 \mathrm{nM} \mathrm{tZ}$ or BA, cotransfected with effector genes, or addition of cell extracts as indicated, normalized to empty vector 
control. Data represent mean values, error bars represent s.d. (A,E) or s.e.m. (B,C,D,F), **p $<0.01$, $* \mathrm{p}<0.05$, ANOVA with Tukey's HSD post hoc test.

\section{Supplementary Materials:}

Materials and Methods

Figures S1-S8

Tables S1-S2

References (30-41) 


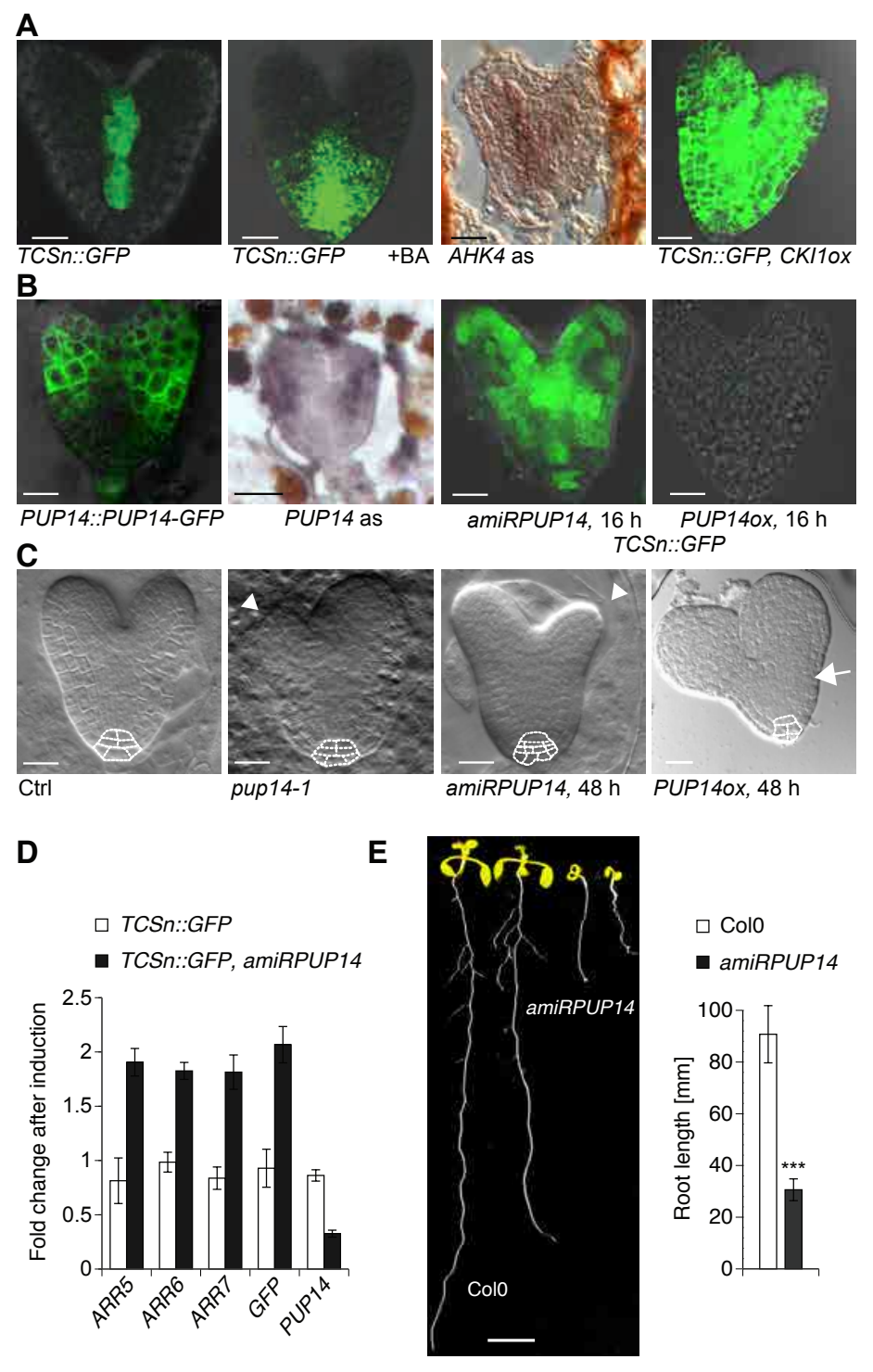

Fig. 1. PUP14 function in embryo and seedlings. 


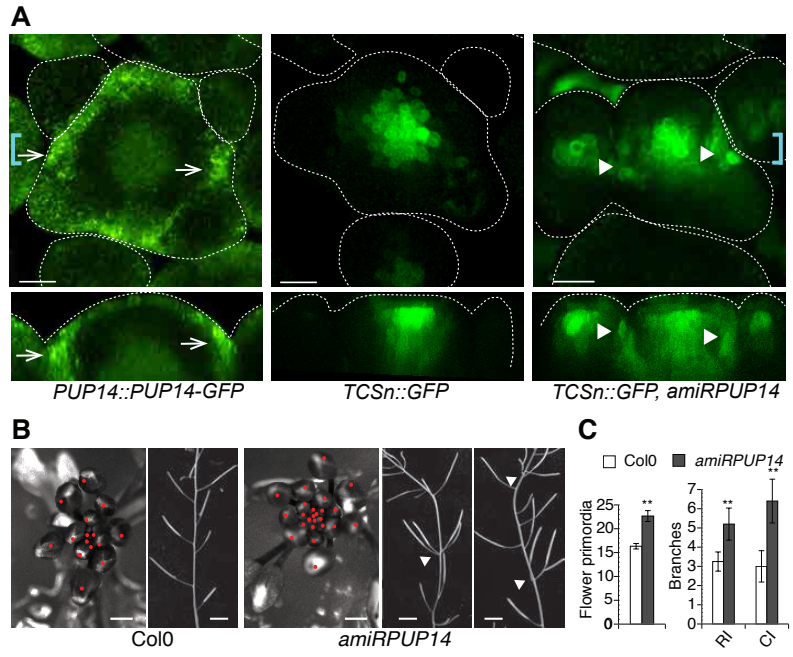

Fig. 2. PUP14 confines the cytokinin response in the SAM. 
A

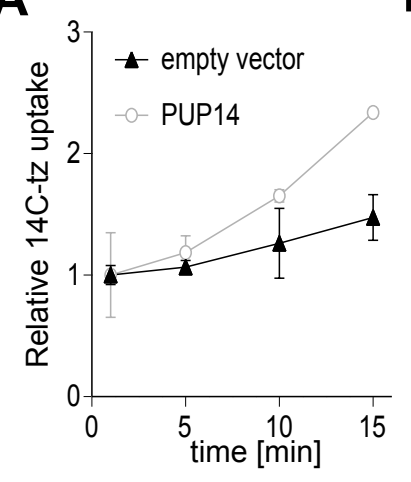

B

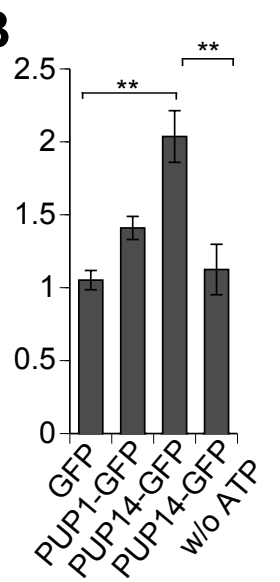

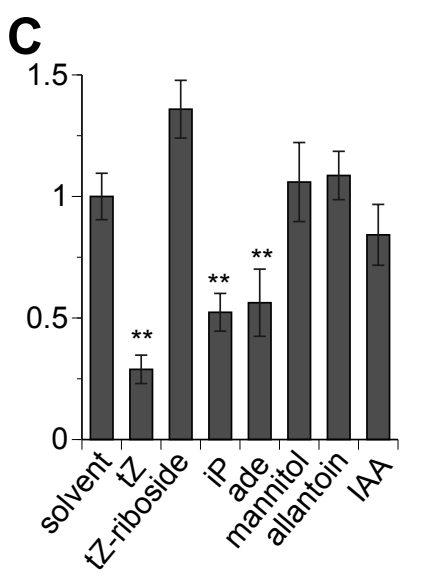

D

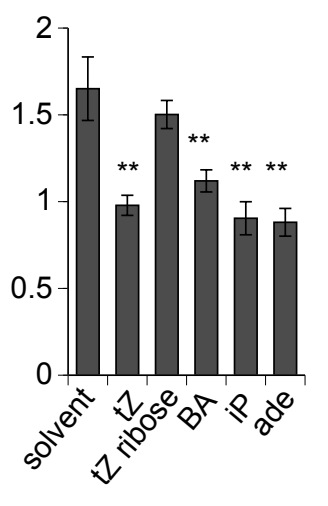

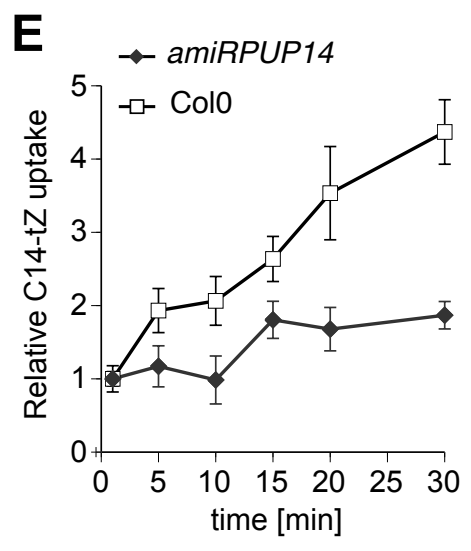

F

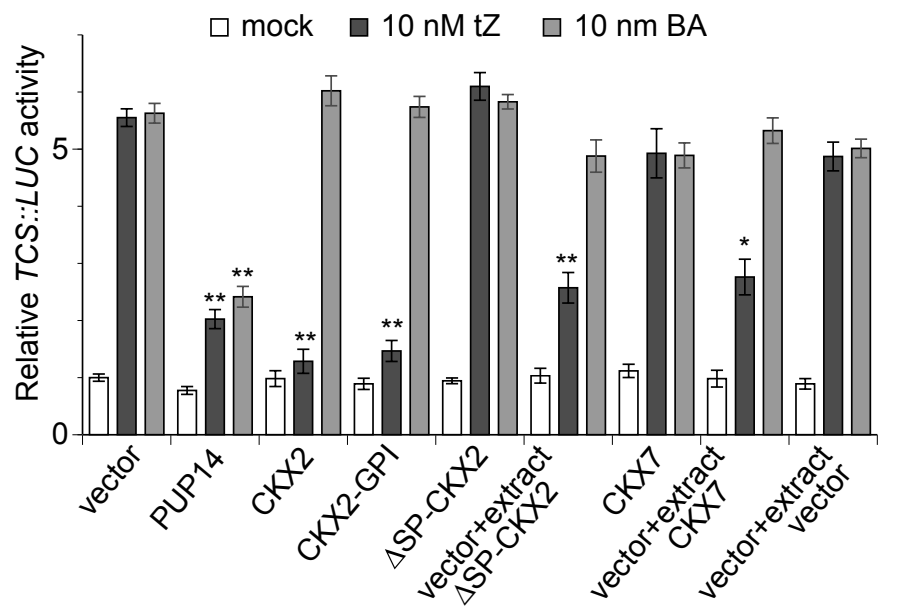

Fig. 3. PUP14 cellular function. 


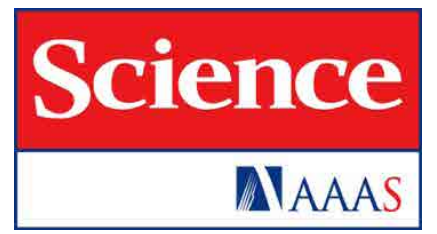

\section{Supplementary Materials for}

\section{Plant Development Regulated by Cytokinin Sinks}

Evelyne Zürcher, Jingchun Liu, Martin di Donato, Markus Geisler \& Bruno Müller correspondence to: bruno.mueller@uzh.ch

\section{This PDF file includes:}

Materials and Methods

SupplementaryText

Figs. S1 to S8

Tables $\mathrm{S} 1$ to $\mathrm{S} 2$ 


\section{Materials and Methods}

Microscopy and live imaging.

RNA in situ hybridization and embryo clearings were observed with a transmission microscope under bright field or differential interference contrast with a 40x lens. For GFP imaging, live embryos or seedlings were mounted in 0.5 strength Murashige and Skoog (MS) liquid medium. SAMs were mounted in warm $0.8 \%$ low-gelling agar dissolved in 0.5 strength liquid MS. Confocal microscope observations were done on a SP2 or SP5 spectral detection confocal microscope (Leica) equipped with a 20x glycerol immersion (seedling root, seeds), 40x oil immersion (SAM) or 63x glycerol immersion lens (female gametophytes, embryos). Images were processed using Imaris (Bitplane, Zurich). Maximum partial projections of equivalent serial sections are shown. Fluorescence levels (TCSn::GFP and DR5::tdTomato) (31) were quantified from maximum intensity projections obtained from 6 embryos using ImageJ (http://rsbweb.nih. gov/ij/). Adult plants were recorded with a DP3 Merrill digital camera (Sigma), and flower primordia with a MZFLII fluorescence stereomicroscope equipped with a DFC 420C digital camera (Leica).

\section{Plant material and growth conditions.}

The ecotype Col0 was used as wild type. Seeds were surface sterilized for $18 \mathrm{~min}$ in $5 \%$ (v/v) bleach and $0.1 \%(\mathrm{v} / \mathrm{v})$ triton-X, washed three times in sterile $\mathrm{ddH}_{2} \mathrm{O}$ and kept in the dark at $4{ }^{\circ} \mathrm{C}$ for a minimum of $2 \mathrm{~d}$ for stratification. Seeds were sown on solid medium, containing 0.5 strength MS, $2 \%(\mathrm{w} / \mathrm{v})$ sucrose, $0.8 \%(\mathrm{w} / \mathrm{v})$ phytagar and $2 \mathrm{mM}$ 2-(N-morpholino)ethanesulfonic acid (MES) $\mathrm{pH} 5.8$, and the appropriate antibiotic or herbicide for selection. Seeds on selection plates were placed into a Percival plant incubator (CU-36L6/D Percival Scientific Inc., Perry IA, USA) with $22^{\circ} \mathrm{C}$ and a $14 / 10 \mathrm{~h}$ light/dark regime with 120 $\mathrm{mmol} \mathrm{m} \mathrm{m}^{-2} \mathrm{~s}^{-1}$. To phenotype seedlings, plants were grown vertically on $12 \mathrm{~cm}$ square plates containing 10 $\mathrm{mL}$ of medium. To prevent desiccation of the plates, $1 \mathrm{~mL}$ of $\mathrm{ddH}_{2} \mathrm{O}$ was added and the plates were sealed with parafilm. Seeds on vertical plates were placed into a Percival plant incubator (CU-36L6 Percival Scientific Inc., Perry IA, USA) with $22^{\circ} \mathrm{C}$ and $12 / 12 \mathrm{~h}$ light/dark cycles with $90 \mathrm{mmol} \mathrm{m}{ }^{-2} \mathrm{~s}^{-1}$. To obtain adult plants, seedlings were transferred to soil and grown at $22{ }^{\circ} \mathrm{C}$ during the day, $20{ }^{\circ} \mathrm{C}$ at night with a $16 / 8$ $\mathrm{h}$ photoperiod.

\section{Plant transformation.}

Plant transformation was performed using Agrobacterium tumefaciens of the GV3101 strain (32). Inducible expression constructs were supertransformed into TCSn::GFP or PUP14::PUP14-GFP transgenic lines.

\section{Ethanol induction of transgenes}

Ethanol was applied as follows to induce expression of amiRPUP14, CKI1, or PUP14 from the ethanol-inducible two-component system (33). For phenotype assessments of seedlings, $1 \mathrm{~mL}$ of $1 \%(\mathrm{v} / \mathrm{v})$ ethanol was added to the bottom of the vertical plates $4 \mathrm{~d}$ after germination. For expression analyses, $7 \mathrm{~d}$ old seedlings grown on vertical plates were transferred to 6-well culture dishes with $3 \mathrm{~mL}$ of liquid medium (0.5 strength MS, $2 \%$ (w/v) sucrose, $2 \mathrm{mM} \mathrm{MES} \mathrm{pH} \mathrm{5.8)} \mathrm{with} \mathrm{and} \mathrm{without} 1 \%$ (v/v) ethanol) for $16-24 \mathrm{~h}$. Dishes were sealed with parafilm. Induction in adult plants was by watering with $1 \%(\mathrm{v} / \mathrm{v})$ ethanol every $4 \mathrm{~d}$ starting from bolting stage as described (33). Embryo inductions were performed as described (4). Controls shown are TCSn::GFP treated with ethanol in parallel to the experimental genotypes. Similar results were obtained with untreated TCSn::GFP, amiRPUP14 plants.

\section{Constructs}

For PUP14::PUP14-GFP, a DNA fragment encompassing the PUP14 locus including the $2.3 \mathrm{~kb}$ upstream sequence was amplified from Col0 genomic DNA by PCR and cloned into the binary vector pCB302 (34) with the enhanced GFP coding sequence allowing for C-terminal fusions, the nopaline synthase 3-prime untranslated region, and an adaptor for ligation-independent cloning (LIC) (Genbank KX510271) (35). For protoplast experiments, $P U P 14, C K X 2$ and $C K X 7$ genomic regions from translational start to stop were amplified from Col0 genomic DNA and annealed to LIC-modified expression vectors (see Table S2) to yield 2x hemagglutinin (HA) (Genbank KX510274), GFP (Genbank KX510273), or glycosyl phosphatidyl inositol (GPI)-anchored GFP C-terminal translational fusions (Genbank KX510275). CKX2-GPI was cloned by restriction digest of $C K X 2-2 H A$ with PstI and StuI and annealing 
of oligonucleotides encoding the GPI-anchor. The artificial microRNA $(a m i R)$ sequences were obtained through the Web MicroRNA Designer (wmd3.weigelworld.org), and assembled by PCR amplification on pRS300 as template as described (12) using LIC-modified primers A and B (see Table S2) to produce fold-back amiR precursors. The fold-back amiR precursors were cloned into the LIC-modified ethanolinducible binary DM7 vector (5). The sequences of the base-pairing nucleotides of amiRPUP14_1 and $\operatorname{amiRPUP} 142$ are TTATTTGCACAAAGTGTTCTG and TGTTGATAGGTATTTGCACGA, respectively. Both amiRPUP14 constructs caused similar phenotypes upon induction. Corresponding target sites in PUP14 are CAGAACAATTTGTGCAAATAC and TTGTGCAAATACCTATCAACA for amiRPUP14_1 and amiRPUP14_2, respectively. Sequence of the base-pairing nucleotides of amiR19/20 is TTAAAACACGTCCTGCGACGA. Target sequences are TCGTAGCAGGACGTGTTTTAT in $P U P 19$ and TCGTAACAGGACGTGTTTTAT in PUP20 amiR-resistant versions of PUP14 (PUP14*) were constructed by site-directed mutagenesis of the amiRPUP14_2 target site to change all codons within the amiR target site in PUP14 to synonymous codons with overall comparable codon usage frequency. The PUP14* encompasses the PUP14 genomic region and was cloned into pCB302 by LIC. Inserts for the 35S::PUP1 and $35 S:: P U P 14$ binary constructs used for expression in microsomes were amplified from Col0 genomic DNA, and cloned into pPLV26 (36) by LIC. For PUP14ox, the PUP14 translated sequence was amplified from genomic Col0 DNA and ligated into LIC-modified DM7 (5). For 35S $>A L C>P U P 14-G F P, P U P 14-$ GFP was amplified from PUP14::PUP14-GFP and ligated into LIC-modified DM7 (5). All constructs were sequenced to ensure no unwanted mutations were introduced. A plasmid list is provided in Table S2.

\section{Protoplast isolation and transfection}

For transient expression experiments, protoplasts of three- to four-week-old wild-type Arabidopsis plants of the Col0 ecotype were isolated as described (37) with the following adaptations: adjusted concentration was $3 \times 10^{5} \mathrm{ml}^{-1}, 35 S:$ renillaLUC was used to normalize for transfection efficiency (38) of TCS::LUC reporter assays, and WI solution was supplemented with $15 \mathrm{mM}$ sucrose. For reporter assays, transfected protoplasts were incubated over night, DCPIP (2,6-Dichloroindophenol sodium salt hydrate) at 5 $\mu \mathrm{M}$ was added as electron acceptor ( 6 ), as well as $\mathrm{tZ}$ or solvent at indicated concentrations, and protoplasts were harvested $60 \mathrm{~min}$ later for LUC measurements. To obtain crude protein extracts, 3 x $10^{5}$ transfected protoplasts were lysed in $200 \mu$ l extraction buffer $(6)$ with $1.2 \%(\mathrm{v} / \mathrm{v})$ plant protease inhibitor mixture (Sigma P9599) and incubated for $5 \mathrm{~min}$ at room temperature. The samples were then centrifuged at 21'000 rcf for $5 \mathrm{~min}$ at room temperature. Supernatants were transferred to a new tube. $25 \mu 1$ were added to $3 \times 10^{4}$ transfected protoplasts. For transport assays, transfections were scaled up according to needs and purified plasmids were transfected in 1:1 ratio between effector and empty plasmid. Transfected protoplasts were cultivated between 12 and $24 \mathrm{hrs}$ at $22{ }^{\circ} \mathrm{C}$ in light $\left(120 \mathrm{mmol} \mathrm{m}^{-2} \mathrm{~s}^{-1}\right)$. Means and standard error of means of at least two independent experiments with three technical replications each are represented.

\section{In situ hybridizations}

mRNA in situ hybridizations were performed as described (4). Probe templates for AHK4 and PUP14 were synthesized by PCR from Col0 genomic DNA using the following primers: T3_AHK4_f: aattaaccctcactaaagGATCATCACCCGCAACTCTC, T7_AHK4_r: taatacgactcactatagGATCAACACTGAACCGTCGTC, T3_PUP14_f: aattaaccctcactaaagATTCTTCAACCACACGCATGAAC, T7_PUP14_r: taatacgactcactatagACCAAAGCTGTTACACACTTACAC. T3 and T7 RNA polymerase promoter sequences are indicated in lower case font.

\section{Transport assays}

For protoplast transport assays, protoplasts were harvested at $100 \mathrm{rcf}$ for $2 \mathrm{~min}$ and re-suspended in percoll solution ( $0.5 \mathrm{M}$ Sorbitol, $1 \mathrm{mM} \mathrm{CaCl}_{2} 20 \mathrm{mM} \mathrm{MES} \mathrm{pH} \mathrm{5.8,} 25 \%$ (v/v) percoll) and mixed with the same volume of glycine betaine solution ( $0.5 \mathrm{M}$ glycine betaine, $1 \mathrm{mM} \mathrm{CaCl}_{2}, 20 \mathrm{mM} \mathrm{MES}$ pH 5.8) containing ${ }^{14} \mathrm{C}$ - labelled $\mathrm{tZ}$ and ${ }^{3} \mathrm{H}_{2} \mathrm{O}$. The final concentration of labelled $\mathrm{tZ}$ was 1 or $2 \mu \mathrm{M}$. For competition studies, unlabeled cold substrate was added in a 100- fold excess. Transport was stopped by centrifugation of samples on a percoll cushion after indicated time points. For scintillation counting, pelleted protoplasts were transferred into $3 \mathrm{~mL}$ of Ultima Gold ${ }^{\mathrm{TM}}$ (PerkinElmer AG, Schwerzenbach, Switzerland) and subjected to $10 \mathrm{~min}$ of disintegration counting of ${ }^{14} \mathrm{C}$ and ${ }^{3} \mathrm{H}$. Three independent replicates of the uptake experiment 
were conducted with similar results, and means with standard deviations from one representative experiment with four technical replications are shown. For competition assays, mean values from three independent experiments with each four technical replications are shown. Indicated relative uptake was calculated as the radioactivity of ${ }^{14} \mathrm{C}$ per radioactivity of ${ }^{3} \mathrm{H}_{2} \mathrm{O}$ normalized to the first time point $(30 \mathrm{~s})$. For seedling transport assays, twelve-day old induced seedlings were transferred to liquid medium containing $0.5 \mathrm{MS}, 2 \%(\mathrm{w} / \mathrm{v}$ ) sucrose, $2 \mathrm{mM}$ MES and vacuum infiltrated for $5 \mathrm{~min}$ and twice $3 \mathrm{~min}$. For each replicate $>10 \mathrm{mg}$ of plant material was used. Radiolabelled $\mathrm{tZ}$ was added to a final concentration of $2 \mu \mathrm{M}$ in $2 \mathrm{~mL}$. Seedlings were washed after indicated time points with excess volumes of cold 0.5 strength MS, $2 \%(\mathrm{w} / \mathrm{v})$ sucrose, $2 \mathrm{mM}$ MES on a Büchner funnel. Seedlings were dried on filter paper and transferred to $1.5 \mathrm{ml}$ tubes containing $800 \mathrm{~mL}$ of $80 \%(\mathrm{v} / \mathrm{v})$ ethanol and heated for $5 \mathrm{~min}$ at $95^{\circ} \mathrm{C}$. Samples were transferred into scintillation vials containing $3 \mathrm{~mL}$ Ultima Gold ${ }^{\mathrm{TM}}$ (PerkinElmer) and subjected to 2 min disintegration counting of ${ }^{14} \mathrm{C}$. Indicated relative uptake was calculated as the radioactivity per fresh weight normalized to the radioactivity per fresh weight at the first time point $(1 \mathrm{~min})$. Mean values from 3 independent experiments with each 4 technical replications are shown. For microsomal uptake experiments, 35S::PUP1, 35S::PUP14 and 35S::GFP were transiently expressed in $N$. benthamiana leaf tissue by Agrobacterium tumefaciens-mediated transfection and microsomes were prepared as described (39). For tZ-uptake experiments, ${ }^{14} \mathrm{C}$-labelled tZ was diluted into transport buffer $(10 \mathrm{mM}$ Tris- $\mathrm{HCl}, 10 \mathrm{mM} \mathrm{MgCl}, 1 \mathrm{mM}$ EDTA, $1 \mathrm{mM}$ DTT, $10 \%$ sucrose, $\mathrm{pH} 7.6$ with or without $5 \mathrm{mM}$ ATP) and added to $300 \mu \mathrm{g}$ of microsomes to yield a final concentration of $1 \mu \mathrm{M}$ labelled tZ. For substrate competition assays unlabelled substrate was included in the transport buffer at a 100 -fold excess. After $10 \mathrm{~s}$ and 4 min of incubation at $20^{\circ} \mathrm{C}$, aliquots of $100 \mu \mathrm{L}$ were vacuum-filtered on Whatman ${ }^{\mathrm{TM}} \mathrm{NC} 45$ filters (GE Healthcare, Little Chalfont, UK) and washed 3 times with $1 \mathrm{~mL}$ cold $\mathrm{ddH}_{2} \mathrm{O}$. Air-dried filters were objected to scintillation counting as described above. Indicated relative uptake was calculated as the radioactivity normalized to the first time point $(10 \mathrm{~s})$. Means and standard error of means of at least four independent experiments with three technical replications each are represented.

\section{qRT PCR analysis}

Quantification of relative gene expression was done by qRT-PCR on an Applied Biosystems 7500 Fast Real- Time PCR System using SYBR ${ }^{\circledR}$ Green PCR Master Mix (Applied Biosystems, Life Technologies Europe B.V., Zug, Switzerland) or SsoAdvanced ${ }^{\mathrm{TM}}$ Universal SYBR $^{\circledR}$ Green Supermix (BioRad Laboratories AG, 1785 Cressier, Switzerland) according to manufacturer's recommendation. Final primer concentrations were $400 \mathrm{nM}$ in a total volume of $20 \mu \mathrm{l}$. The relative values of the transcripts were normalized to EUKARYOTIC TRANSLATION INITIATION FACTOR 4A (eIF4A, At3G13920) levels or to At2G32170. Fold changes were calculated by the $2^{-\Delta \Delta \mathrm{Ct}}$ method (40). Means and standard error of means of at least three independent experiments with three technical replications each are represented. 


\section{A}

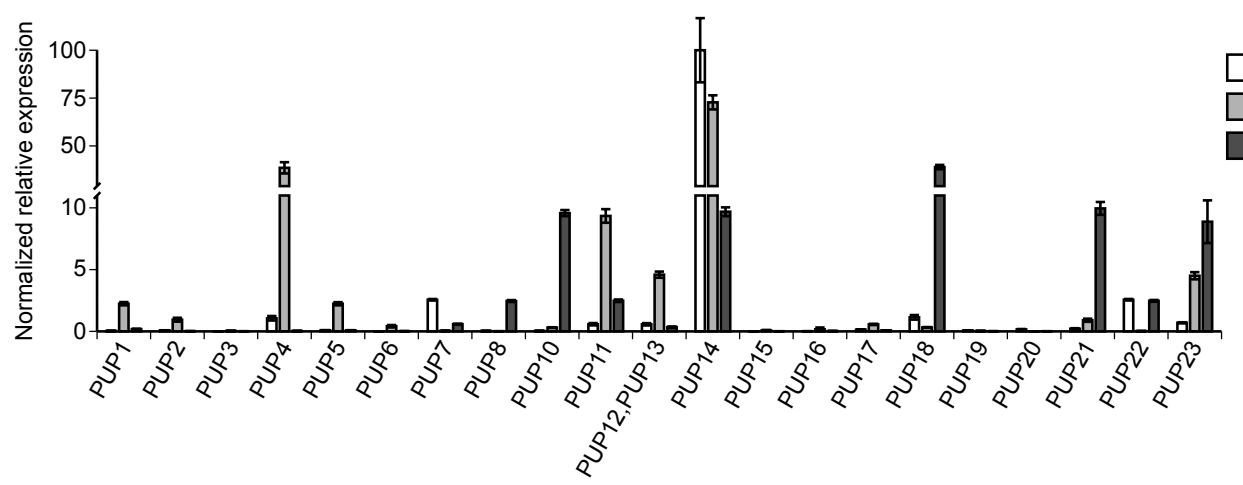

B

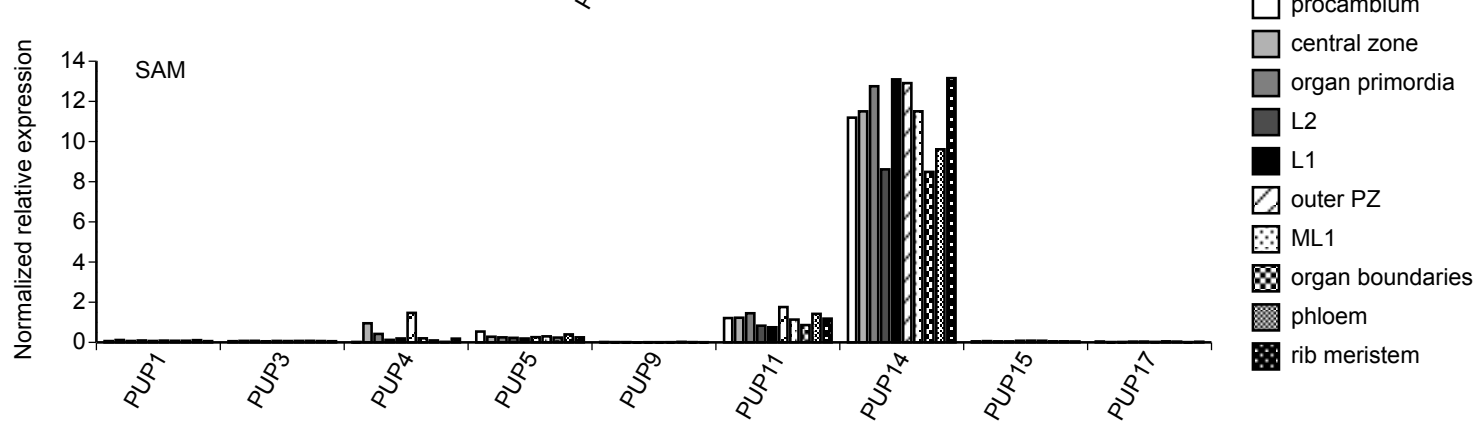

C
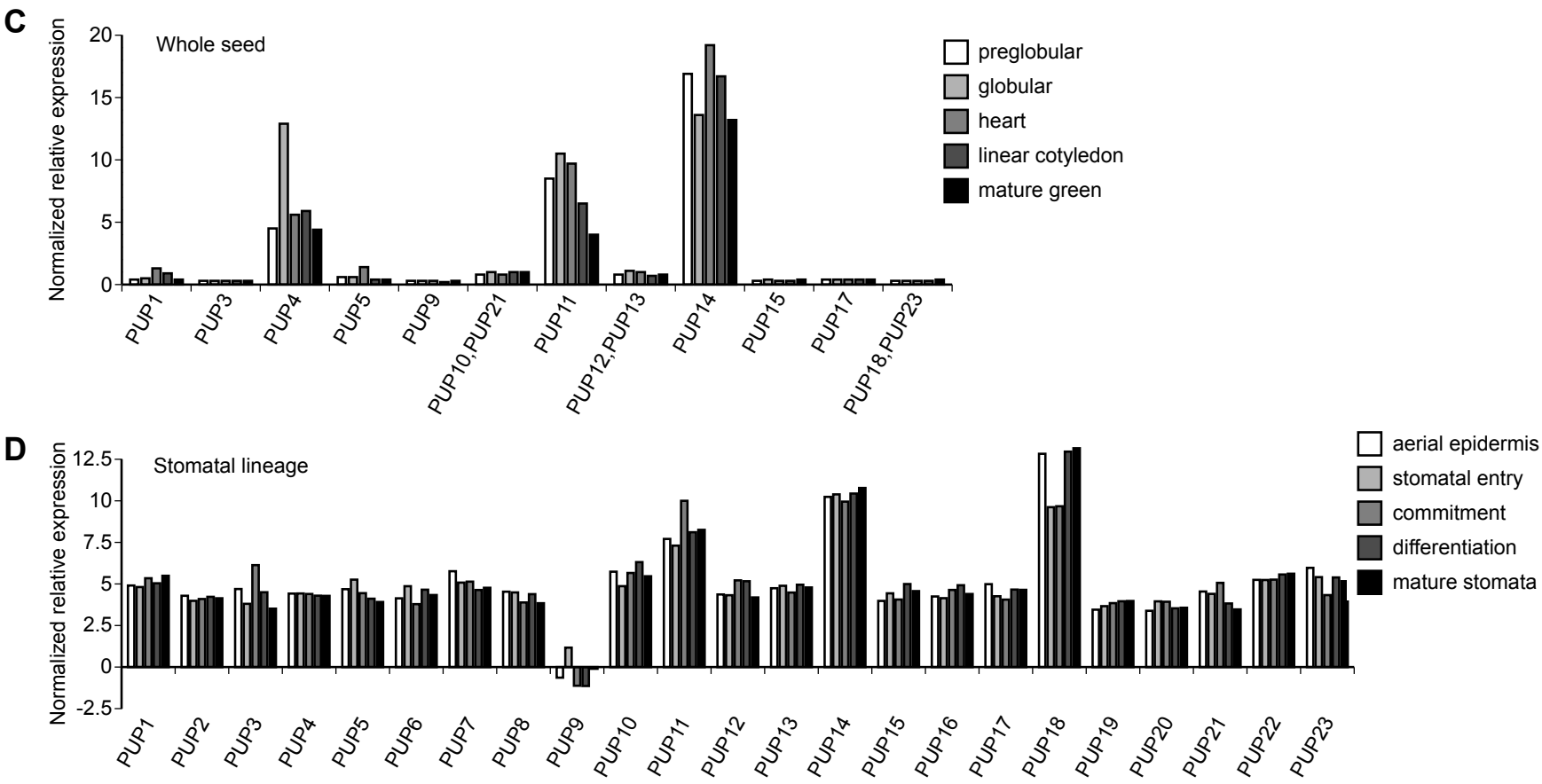

Fig. S1 Conspicuous PUP14 expression in different developmental contexts.

(A) Transcription profile of all PUP family members in seedlings, embryos and mesophyll protoplasts determined by qRT-PCR. (B) Cell type-specific ATH1-based microarray dataset of PUPs in the SAM. L1 = layer 1 in SAM, L2 = layer 2, ML1 = layer 1 in meristem and in differentiating organs, PZ = peripheral zone (11). (C) ATH1 based microarray dataset of PUPs during seed development (10) (D) Stage-specific RNAseq dataset of PUPs in stomatal lineage (9). PUP AGI identifying numbers: PUP1, AT1G28230; PUP2, AT2G33750; PUP3, AT1G28220; PUP4, AT1G30840; PUP5, AT2G24220; PUP6, AT4G18190; PUP7, AT4G18197; PUP8, AT4G18195; PUP9, AT1G18220; PUP10, AT4G18210; PUP11, AT1G44750; PUP12, AT5G41160; PUP13, AT4G08700; PUP14, AT1G19770; PUP15, AT1G75470; PUP16, AT1G09860; PUP17, AT1G57943; PUP18, AT1G57990; PUP19, AT1G47603; PUP20, AT1G47590; PUP21, AT4G18220; PUP22, AT4G18205; PUP23, AT1G57980. 
A

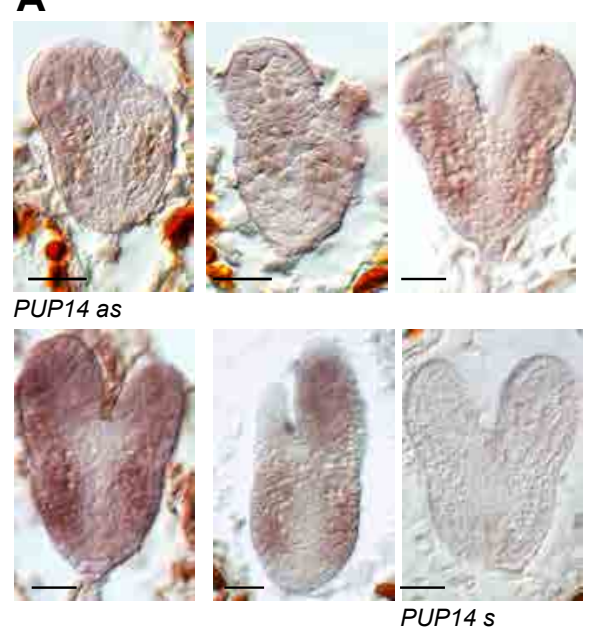

B
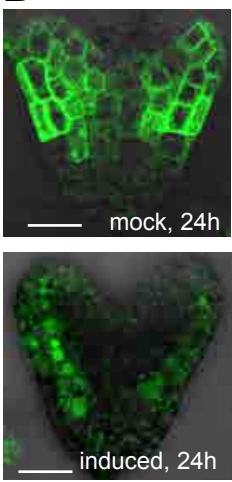

amiRPUP14

PUP14::PUP14-GFP
C

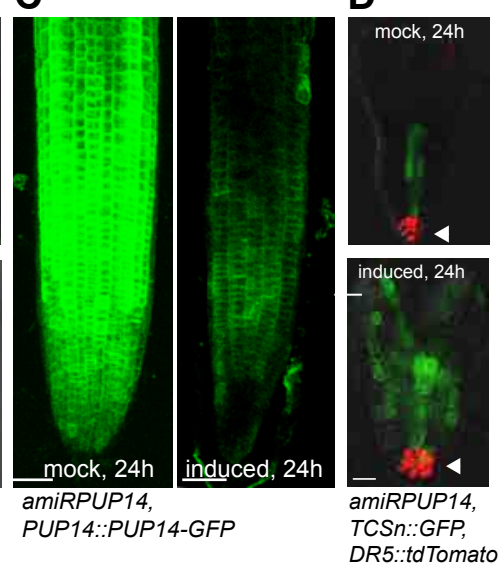

$E$

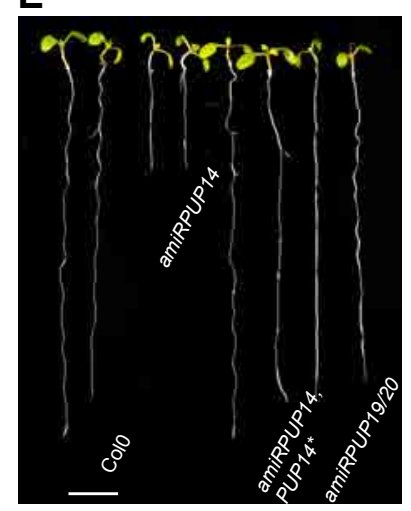

$\mathbf{F}$

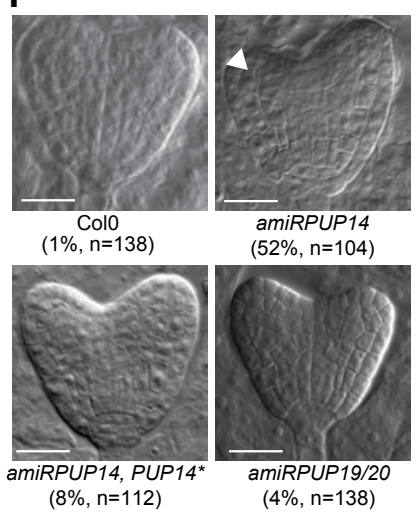

G

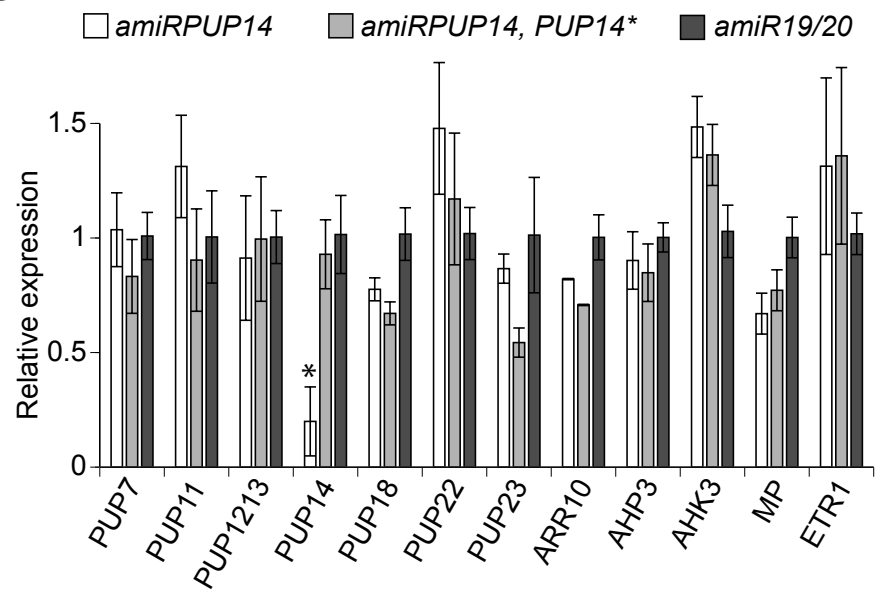

Fig. S2 amiRPUP14-induced phenotypes are specific to PUP14.

(A) Localization of PUP14 mRNA in heart and torpedo stage embryos by in situ hybridization. First five panels show antisense (as) probe, last panel sense (s) control. (B,C) Decreased PUP14-GFP levels in (B) heart-stage embryos and (C) the seedling root tip after $24 \mathrm{~h}$ of amiRPUP14 induction compared to mock, $\mathrm{n}=6$. (D) amiRPUP14 affects cytokinin but not auxin response, as shown by unchanged DR $5:$ :tdTomato (31) expression in amiRPUP14-induced embryos (arrowheads). Relative fluorescence of tdTomato not affected (unpaired t-test, $\mathrm{p}<0.01, \mathrm{n}=5$ ). (E) Comparison of root growth in Col0, amiRPUP14, PUP14*complemented and amiRPUP19/20 seedlings after ethanol induction. (F) Comparison of ethanol-treated embryos of Col0, amiRPUP14, PUP14*-complemented and amiRPUP19/20 seedlings. (G) Relative transcript levels of PUP and unrelated genes in amiRPUP14; amiRPUP14, PUP14* seedlings compared to amiRPUP19/20, all ethanol-treated, error bars denote s.e.m. $* \mathrm{P}<0.05$ with one-way analysis of variance with Tukey' HSD post hoc test. Scale bars (A,B,D,F) $20 \mu \mathrm{m},(C) 50 \mu \mathrm{m}$, (E) $1 \mathrm{~cm}$. 
A

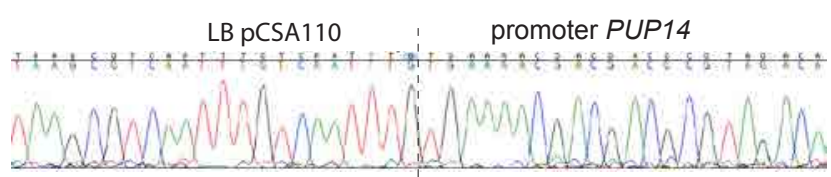

pup14-1, SAIL_93b_F05

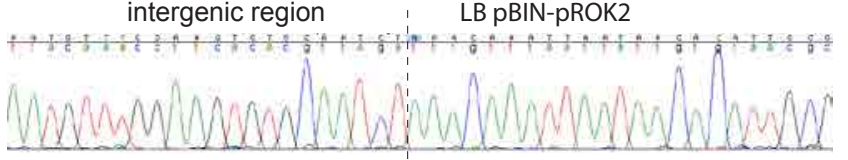

SALK_004230C

$\underline{100 \mathrm{bp}}$

B

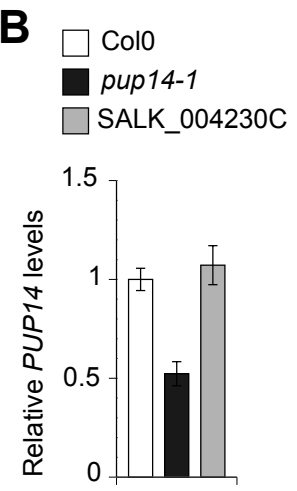

C

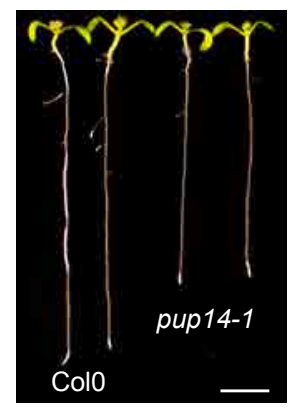

D

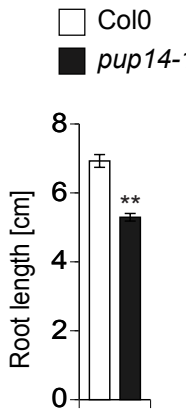

E

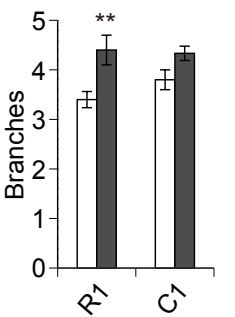

$\mathbf{F}$
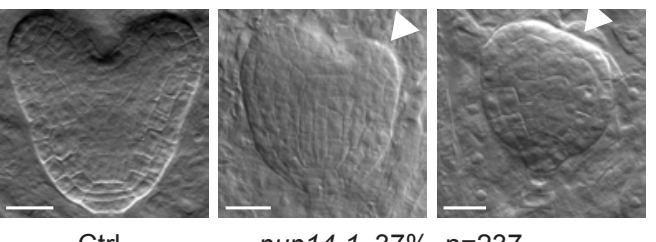

pup $14-1,37 \%, \mathrm{n}=237$

Fig. S3 T-DNA insertion lines at PUP14 locus.

(A) Sequences of insertion sites of pup14-1 (SAIL_93b_F03) and SALK_004230C T-DNA insertion lines and schematic representation of insertion sites on gene level. (B) PUP14 transcript levels in Col0, pup 14-1, SALK_004230C. (C) Root phenotype of pup14-1 compared to Col0. (D) Quantification of root length of pup 14-1 compared to Col0. (E) Comparison of branch numbers, $\mathrm{RI}=$ rosette branches, $\mathrm{CI}=$ primary cauline branches, $\mathrm{n}=6, * * \mathrm{P}<0.01$ one-sided $t$ test. (F) pup 14-1 embryos show morphological defects in cotyledons and root meristems compared to non-affected siblings from the same silique. Error bars denote s.d. Scale bars $1 \mathrm{~cm}(\mathrm{C}), 20 \mu \mathrm{m}(\mathrm{F})$. 
A

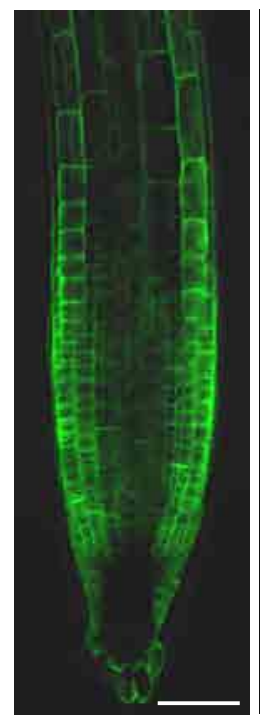

PUP14::

PUP14-GFP

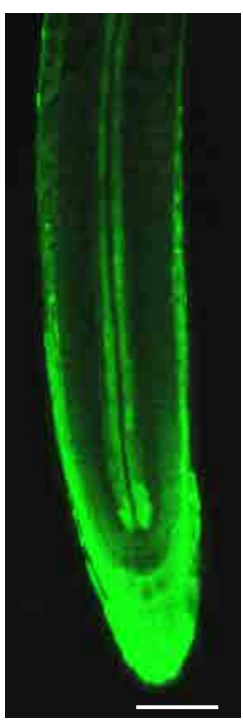

TCSn::GFP

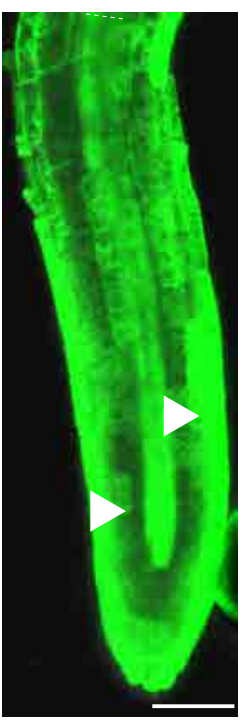

TCSn::GFP, amiRPUP14
B

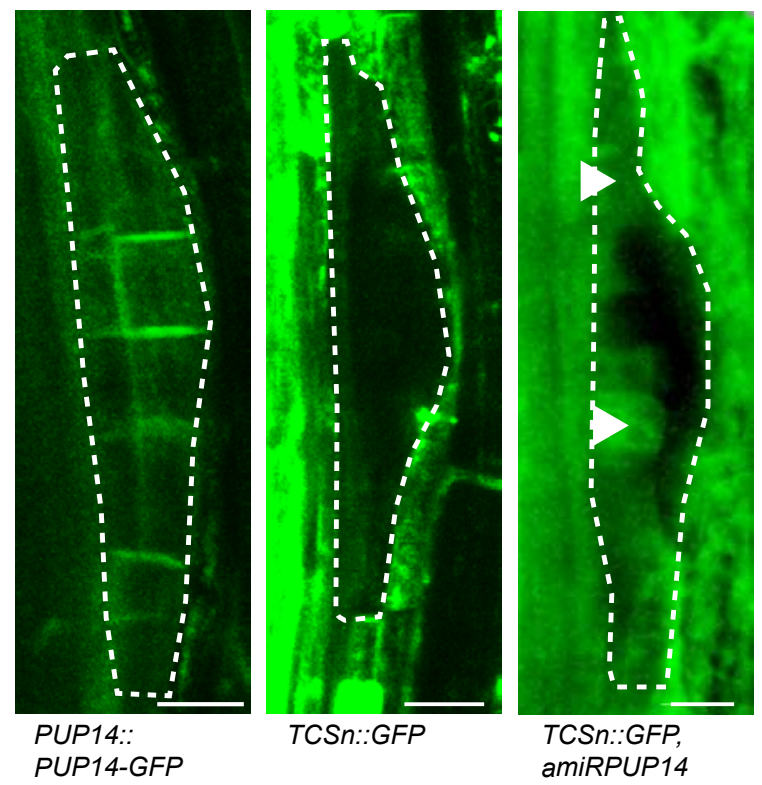

Fig. S4 PUP14 confines the cytokinin response in seedling roots.

(A) PUP14::PUP14-GFP in main root. TCSn::GFP in the main root. Ectopic TCSn::GFP after 16 $\mathrm{h}$ amiRPUP14 induction (arrowheads) ( $80 \%$ of roots, $\mathrm{n}=15)$ roots. (B) LRP, denoted by dotted line. PUP14::PUP14-GFP, TCSn::GFP, ectopic TCSn::GFP (arrowheads) $16 \mathrm{~h}$ after amiRPUP14 induction. Scale bars (A) $50 \mu \mathrm{m}$, (B) $10 \mu \mathrm{m}$. 
A
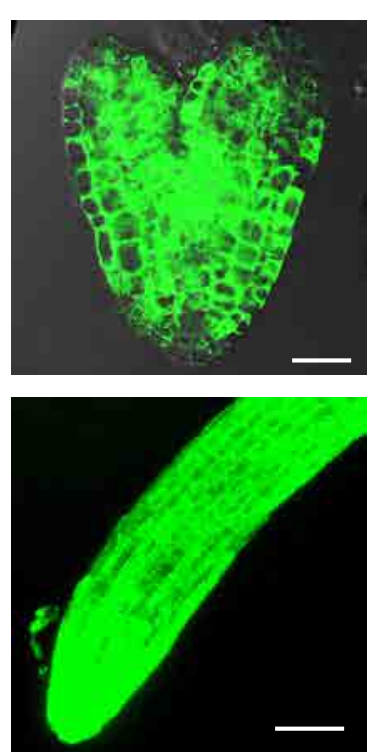

$35 S>A L C>G F P$
B
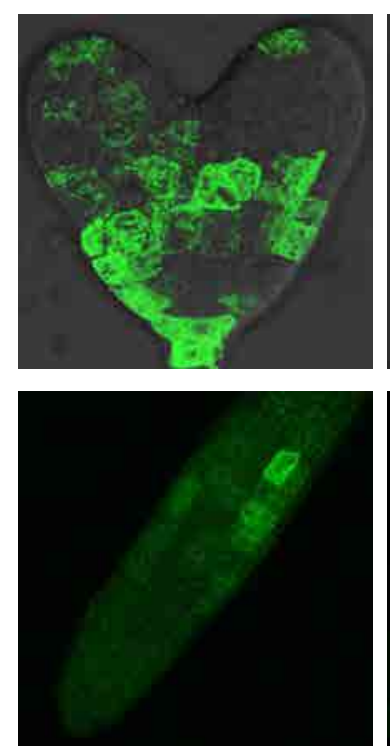

35S $>A L C>P U P 14-G F P$
C
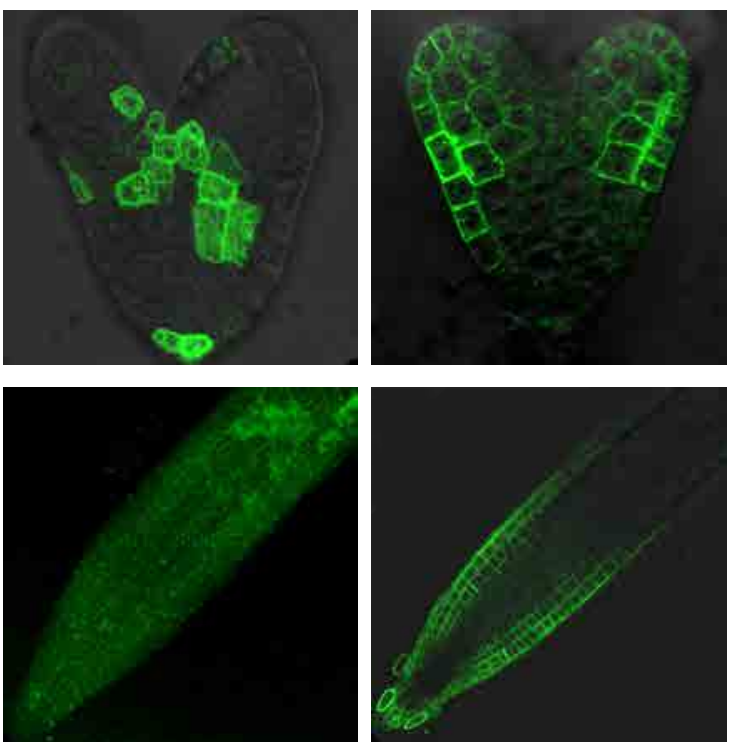

PUP14::PUP14-GFP

Fig. S5 Ectopic PUP14-GFP expression is not well tolerated by plants.

Heart-stage embryos (top panels) and main root apices of $7 \mathrm{~d}$ old seedlings (bottom panels) showing (A) 24 h ethanol-induced $35 S>A L C>G F P$ overexpression, (B) 24 h ethanol-induced $35 S>A L C>P U P 14$ GFP overexpression and (C) PUP 14::PUP14-GFP expression. Scale bars $20 \mu \mathrm{m}$ (top panel), $50 \mu \mathrm{m}$ (bottom panel). 

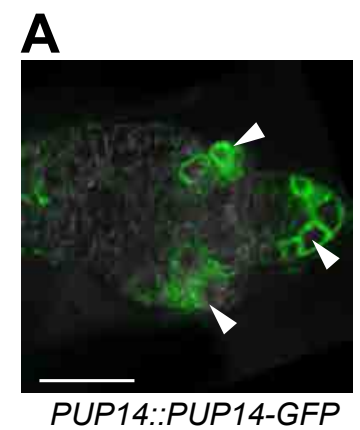

B

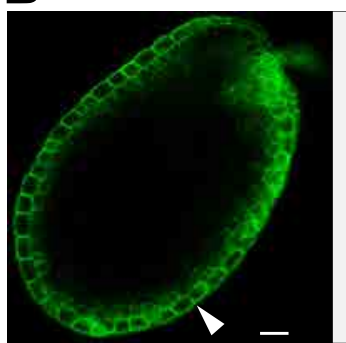

PUP14::PUP14-GFP

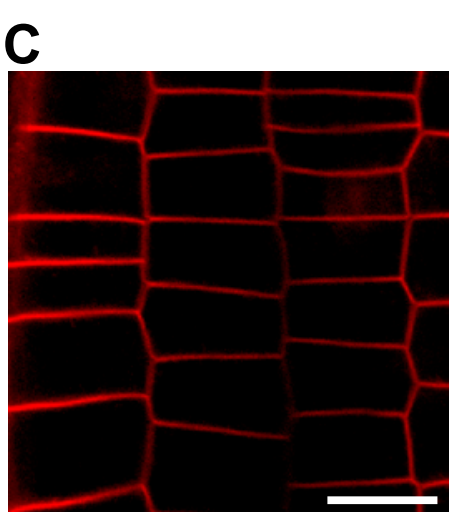

FM4-64

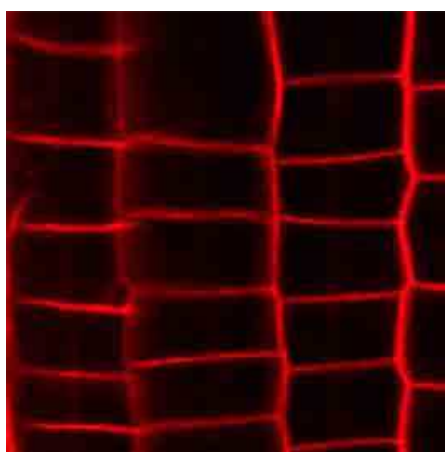

FM4-64

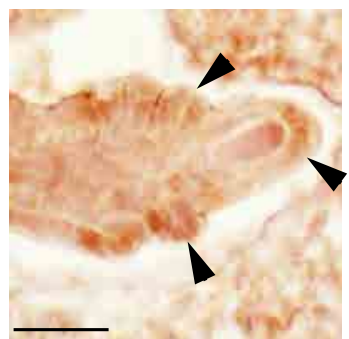

PUP14 as

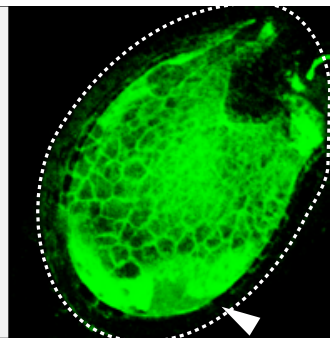

TCSn::GFP

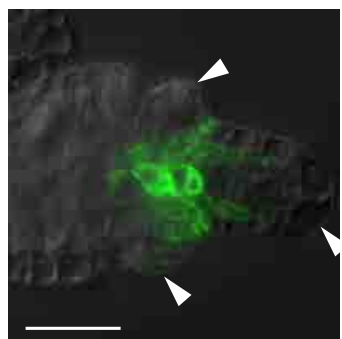

TCSn::GFP

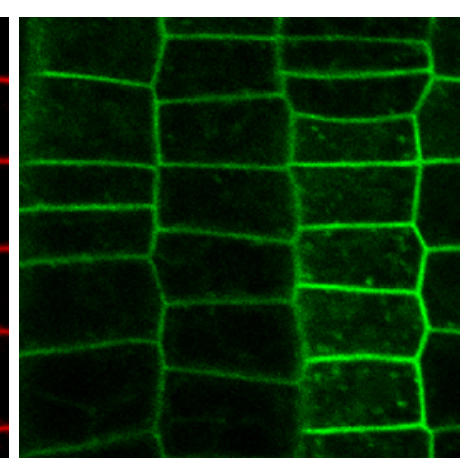

PUP14::PUP14-GFP

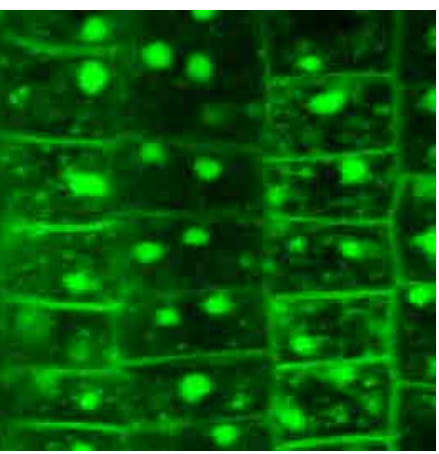

PUP14::PUP14-GFP

$+25 \mu \mathrm{M}$ BFA 30 min

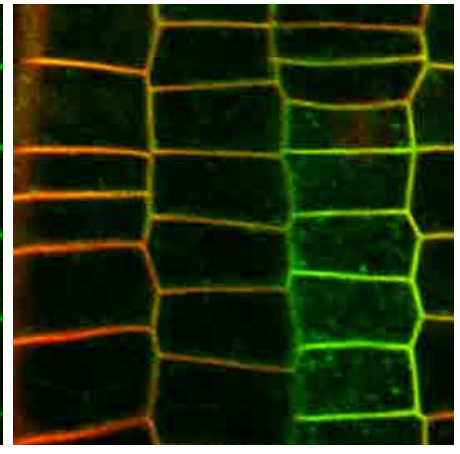

merge

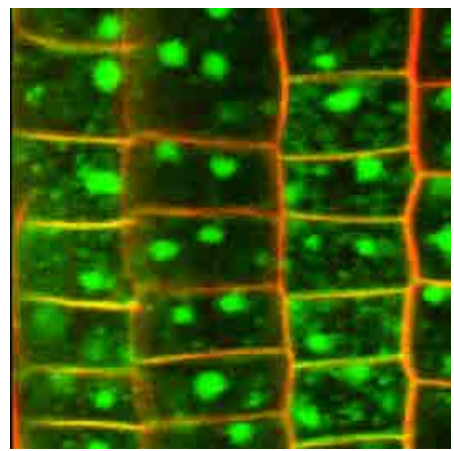

merge

\section{Fig. S6 PUP14 expression and subcellular localization.}

$(\mathrm{A}, \mathrm{B})$ PUP14 expression and cytokinin signaling output in (A) the developing ovule primordium and (B) the seed, detected by reporter transgenes as indicated. In addition, PUP14 mRNA is detected by in situ hybridization with PUP14 as RNA probe in (A). Dotted lines delimit the seed coat. Arrowheads indicate peak PUP14-GFP expression levels. (C) PUP14-GFP subcellular localization and trafficking in main root. PUP14-GFP colocalization with plasma membrane marker dye FM4-64 (top panels). Addition of the fungal toxin brefeldin A (BFA) causes accumulation of PUP14-GFP signal in vesicles (bottom panels). Scale bars (A,C) $10 \mu \mathrm{m}$, (B) $20 \mu \mathrm{m}$. 


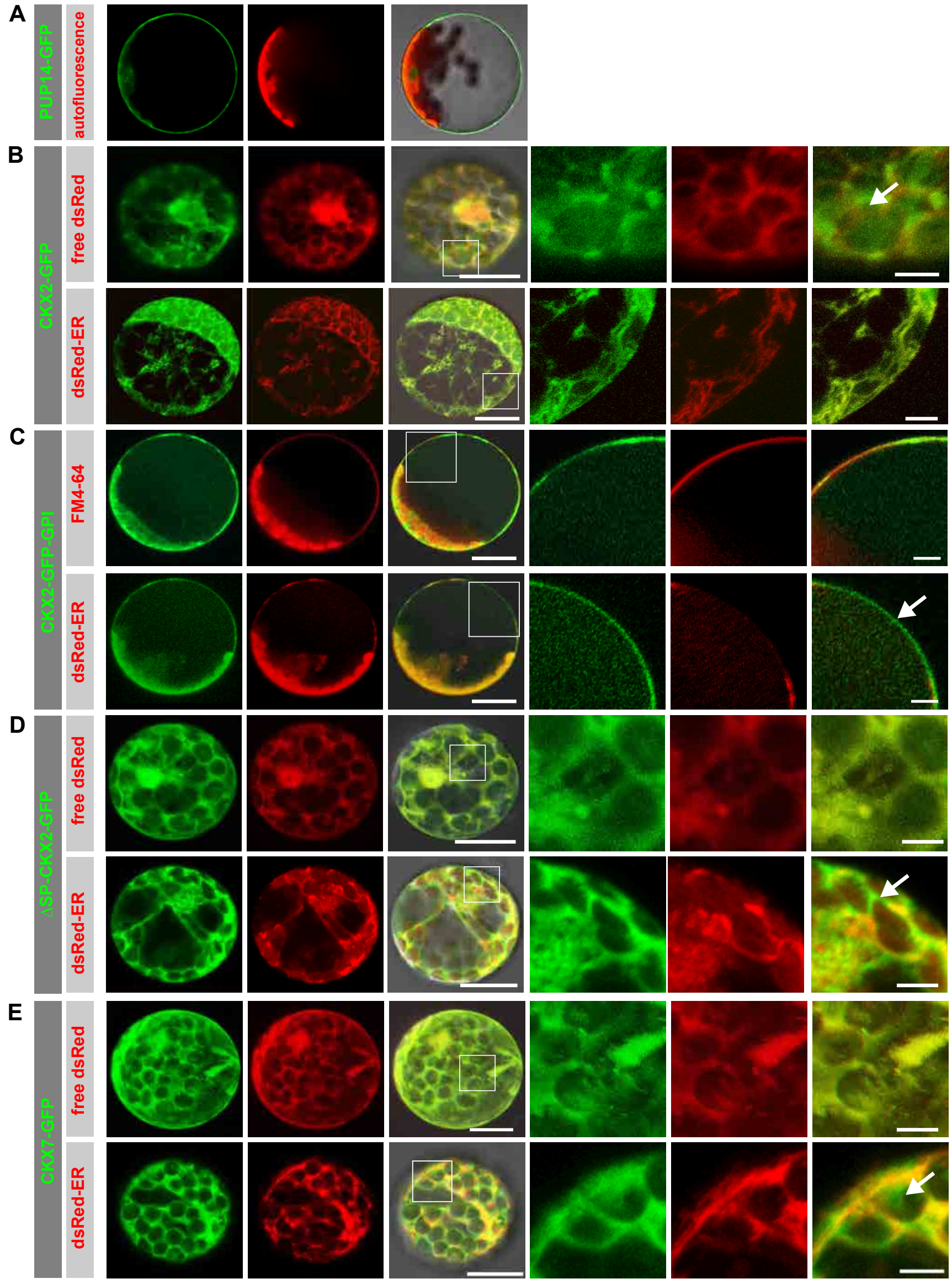

Fig. S7 Subcellular co-localizations of differentially targeted CKX-GFP variants in protoplasts.

(A) Localization of PUP14-GFP and (B-E) co-localization of GFP-tagged $C K X$ gene products with subcellular markers in protoplasts as indicated. ER labelling with ER-dsRed, labeling of cytoplasm with free dsRed (41), and plasma membrane staining with the lipophilic dye FM4-64. Boxes indicate areas of the enlargement shown to the right. Arrows point to non-overlapping signals. Scale bars $20 \mu \mathrm{m}$, in enlargements 5 $\mu \mathrm{m}$. 


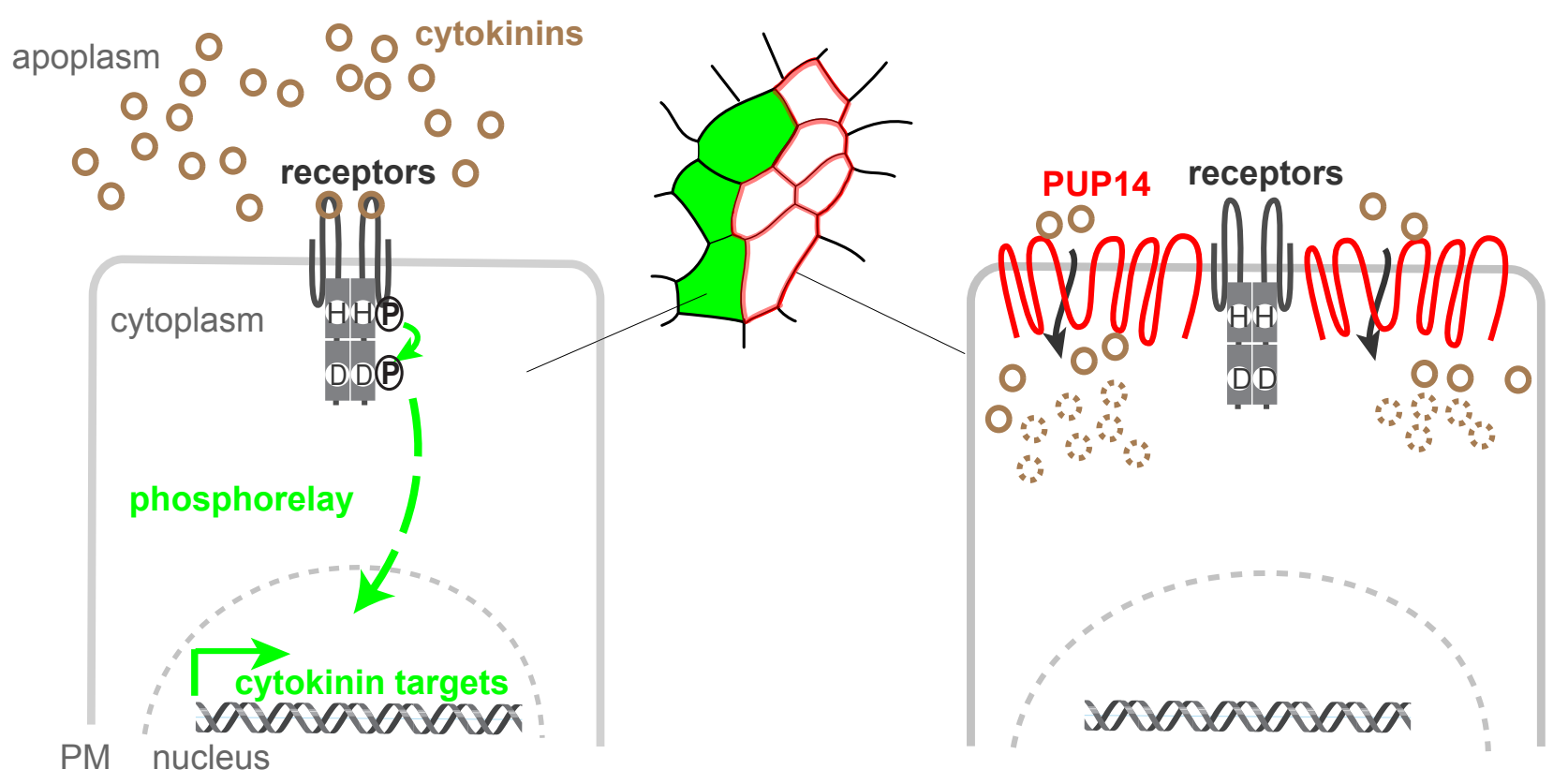

\section{Fig. S8 Model of PUP14 function in cytokinin signaling.}

PUP14 (red) causes the translocation of apoplastic cytokinins to the cytosol, where they are converted to inactive forms (dotted circles). This results in reduced binding to plasma membrane-localized cytokinin receptors, and consequently reduced signaling activation (green denotes cytokinin signaling activity). PM: plasma membrane. 


\begin{tabular}{|c|c|c|}
\hline Target gene & Forward primer sequence 5 ' to $3^{\prime}$ & Reverse primer sequence $5^{\circ}$ to $3^{\prime}$ \\
\hline elF4a & TCATAGATCTGGTCCTTGAAACC & GGCAGTCTCTTCGTGCTGAC \\
\hline ARR5 & GGTTGGATTTGAGGATCTGAAG & TCCAGTCATCCCAGGCATAG \\
\hline ARR6 & TTGCCTCGTATTGATAGATGTCTT & CCGAGAGTTTTACCGGCTTC \\
\hline ARR7 & AGATTAAGGAATCTTCAGCATTCAG & CTGCTAGCTTCACCGGTTTC \\
\hline mGFP & TCAAGGACGACGGGAACTAC & ATCCTGTTGACGAGGGTGTC \\
\hline PUP1 & TGTTTCCGGGAGAAGTTTCA & CGGATTTAAACTCGCCGTAG \\
\hline PUP2 & TCTGTGCATCGTCTCTGGTC & TCTCCTGGAAGCAAATGACG \\
\hline PUP3 & AATACCCGAGACGAGAGACG & CGTCTCTCGTCTCGGGTATT \\
\hline PUP4 & ACCGGAGGTATCTGCATGAC & САСТCCACCAAACACGTCAC \\
\hline PUP5 & TGCAGTCACGTTTCAACTGG & TGACTGTGGATGCCAGAAAC \\
\hline PUPG & TGCCTGTTCTTGCTGTTGTC & TCTTGGTCTTCTTCTGGCTTTC \\
\hline PUP7 & TTTAGCTATCTGCGGCTTCC & GTGTGACCTTCCTCAACAGG \\
\hline PUPB & GTCGTGGGACTGATCTTTGAG & GCAATCCCACAGCAGTTATG \\
\hline PUP10 & ACCCACCAGAAGCAGAAGAG & GTAAACTGCGGGACAGCATC \\
\hline PUP11 & TCGACGTATTCGCTCATTTG & GCGGAGAACGACAAGAGAAC \\
\hline PUP12/13 & AGGTTAAGATGGTGGCGATG & TGAGCTTCTCGTGCTCTTTG \\
\hline PUP14 & TCTGTTTCGAGCGTGTTGTC & GCGCTTAAGACGGCAGTAAC \\
\hline PUP15 & GCAGCTGCTCTTAGCGTCTC & TTGTGGATTGGTCATCATCG \\
\hline PUP16 & GTCCGGTTTATTCGCTGATG & AGCACCTCTTCTCTGCCAAC \\
\hline PUP17 & GGCCTAGAATTGGTGCTTTG & TTTGGTTAAGTTCCGCCATC \\
\hline PUP18 & TGCTTTATGTTTCGGGTGTG & CAAAGCCACAAGTGGTGAAG \\
\hline PUP19 & CTGGTAGCTGGGATTCTTGG & AGTTGTTTGGCTTTGGCTTG \\
\hline PUP2O & TTTAGGGCTTGTGGGTCTTG & GCTCCTCCCTTAAACCATCC \\
\hline PUP21 & TTGCACAGGACTGATCTTCG & TGACAGCCAGGATAGGAACC \\
\hline PUP22 & ATCTTGACTTTGGCCTCAGC & GCAGTCCCACAGCAGTTATG \\
\hline PUP23 & TGTGTGCTTCACCACTTATGG & AGCACCAATTCTAGGCCAAC \\
\hline AT2G32170 & TGCTTTTTCATCGACACTGC & CCATATGTGTCCGCAAAATG \\
\hline АНКЗ & GGTGGAGTTGGCAGTATACATATC & CGAAACCTCCCAGGATCAC \\
\hline AHP3 & TCTCAGAACTATGAAGGGTGTGTG & AATGTCTTGTACTCAATATCCACTTGC \\
\hline ARR10 & GACACAGGAACAGAGCCAATC & TATGCATGTTCCGAGTGAGC \\
\hline ETR1 & GAGAAGCTCGGGTGGTAGTG & TTTCCAAGACCATCGCTCTC \\
\hline ARF5/MP & СССТTCTTCACTCATCTGCTG & TCCATGGGAAGAGTTTGTGG \\
\hline
\end{tabular}

Table S1.

qRT-PCR primer sequences used in this study. 


\begin{tabular}{|c|c|c|c|c|c|c|}
\hline \multirow{2}{*}{ Name } & \multirow{2}{*}{ Purpose } & \multirow{2}{*}{ Parent vector } & \multirow{2}{*}{$\begin{array}{c}\text { Selection } \\
\text { (bacteria/plants) }\end{array}$} & \multicolumn{3}{|c|}{ Insert } \\
\hline & & & & Primer name & Sequence $5^{\prime}$ to $3^{\prime}$ & Template \\
\hline \multirow{6}{*}{$35 S>A L C>a m i R P U P 14 \_1$} & \multirow{6}{*}{$\begin{array}{c}\text { Ethanol-inducible binary } \\
\text { vector with amiR specific } \\
\text { for PUP14 (variant 1) }\end{array}$} & \multirow{6}{*}{$D M 7-L I C(5)$} & \multirow{6}{*}{ Kan/Kan } & LIC-OLIGO_A & $\begin{array}{l}\text { tagttggaatgggttcgaaCGACGTTGTAAAACGACGG- } \\
\text { CCAG }\end{array}$ & \multirow{6}{*}{ pRS300 } \\
\hline & & & & LIC-OLIGO_B & $\begin{array}{l}\text { ttatggagttgggttcgaaCTCGGAATTAACCCT- } \\
\text { CACTAAAGG }\end{array}$ & \\
\hline & & & & amiR_14_1_l & gaTTATTTGCACAAAGTGTTCTGtctctctttgtattcc & \\
\hline & & & & amiR_14_1_II & gaCAGAACACTTTGTGCAAATAAtcaaagagaatcaatga & \\
\hline & & & & amiR_14_1_III & gaCAAAACACTTTGTCCAAATATtcacaggtcgtgatatg & \\
\hline & & & & amiR_14_1_IV & gaATATTTGGACAAAGTGTTTTGtctacatatatattcct & \\
\hline \multirow{6}{*}{ 35S>ALC>amiRPUP14_2 } & \multirow{6}{*}{$\begin{array}{c}\text { Ethanol-inducible binary } \\
\text { vector with amiR specific } \\
\text { for PUP14 (variant 2) }\end{array}$} & \multirow{6}{*}{$D M 7-L I C(5)$} & \multirow{6}{*}{ Kan/Kan } & LIC-OLIGO_A & $\begin{array}{l}\text { tagttggaatgggttcgaaCGACGTTGTAAAACGACGG- } \\
\text { CCAG }\end{array}$ & \multirow{6}{*}{ pRS300 } \\
\hline & & & & LIC-OLIGO_B & $\begin{array}{l}\text { ttatggagttgggttcgaaCTCGGAATTAACCCT- } \\
\text { CACTAAAGG }\end{array}$ & \\
\hline & & & & amiR_PUP14_2_I & gaTGTTGATAGGTATTTGCACGAtctctctttgtattcc & \\
\hline & & & & amiR_PUP14_2_II & gaTCGTGCAAATACCTATCAACAtcaaagagaatcaatga & \\
\hline & & & & amiR_PUP14_2_III & I gaTCATGCAAATACCAATCAACTtcacaggtcgtgatatg & \\
\hline & & & & amiR_PUP14_2_IV & / gaAGTTGATTGGTATTTGCATGAtctacatatatattcct & \\
\hline \multirow{6}{*}{$35 S>A L C>a m i R P U P 19 / 20$} & \multirow{6}{*}{$\begin{array}{c}\text { Ethanol-inducible binary } \\
\text { vector with amiR specific } \\
\text { for PUP19 and PUP20 }\end{array}$} & \multirow{6}{*}{$D M 7-L I C(5)$} & \multirow{6}{*}{ Kan/Kan } & LIC-OLIGO_A & $\begin{array}{l}\text { tagttggaatgggttcgaaCGACGTTGTAAAACGACGG- } \\
\text { CCAG }\end{array}$ & \multirow{6}{*}{ pRS300 } \\
\hline & & & & LIC-OLIGO_B & $\begin{array}{l}\text { ttatggagttgggttcgaaCTCGGAATTAACCCT- } \\
\text { CACTAAAGG }\end{array}$ & \\
\hline & & & & amiR_19/20_I & gaTTAAAACACGTCCTGCGACGAtctctctttgtattcc & \\
\hline & & & & amiR_19/20_II & $\begin{array}{l}\text { gaTCGTCGCAGGACGTGTTTTAAtcaaagagaat- } \\
\text { caatga }\end{array}$ & \\
\hline & & & & amiR_19/20_III & gaTCATCGCAGGACGAGTTTTATtcacaggtcgtgatatg & \\
\hline & & & & amiR_19/20_IV & gaATAAAACTCGTCCTGCGATGAtctacatatatattcct & \\
\hline \multirow{2}{*}{ PUP14::PUP14-GFP } & \multirow{2}{*}{ Reporter } & \multirow{2}{*}{$\begin{array}{c}\text { pCB302 LIC } \\
\text { GFP (Genbank } \\
\text { KX510271) }\end{array}$} & \multirow{2}{*}{ Kan/Basta } & PUP14_LICF-F & $\begin{array}{l}\text { tagttggaatgggttcgaGCTTCTGCAGTGAAA- } \\
\text { GATGTGTT }\end{array}$ & \multirow{2}{*}{ Col0 genomic } \\
\hline & & & & $\begin{array}{l}\text { PUP14_LIC_- } \\
\text { GFP302_R }\end{array}$ & $\begin{array}{l}\text { tattggagttgggttcgaaTAAGCCATACGATTGTCTT- } \\
\text { TGTG }\end{array}$ & \\
\hline \multirow{2}{*}{$\begin{array}{l}\text { hbt::PUP14-GFP; } \\
\text { 35S::PUP14 }\end{array}$} & \multirow{2}{*}{$\begin{array}{l}\text { Protoplast expression } \\
\text { vector; binary vector } \\
\text { for expression in } \\
\text { microsomes }\end{array}$} & hbt::LIC-GFP; & Amn. Kan/Kan & PUP14_LIC_F & $\begin{array}{l}\text { tagttggaatgggttcgaATCCATGGCTCAGAATCAA- } \\
\text { CAAC }\end{array}$ & Coln aenomic \\
\hline & & pPLV26 (34) & 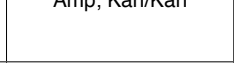 & PUP14_LIC_R & $\begin{array}{l}\text { ttatggagttgggttcgaaATAAGCCATACGATTGTCTT- } \\
\text { TGTG }\end{array}$ & 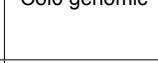 \\
\hline PUP14 nCR302 & PUP14 genomic region & pCB302 LIC & Kan/Rasta & PUP14_LICF-F & $\begin{array}{l}\text { tagttggaatgggttcgaGCTTCTGCAGTGAAA- } \\
\text { GATGTGTT }\end{array}$ & Coln genomic \\
\hline 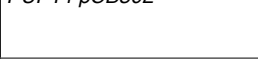 & in binary vector & KX510272) & 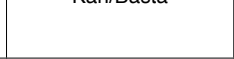 & $\begin{array}{l}\text { PUP14__ } \\
\text { LIC3prime_R }\end{array}$ & ttatggagttgggttcgaaGCACACTTCCAAACATTTTCA & \\
\hline PUP14* & amiRPUP14_2-resistant & PUP14 חCR30? & Kan/Bacta & amiR14_2R* F & $\begin{array}{l}\text { CTCTGTTTCTTTTTGCAGAACAATTTGTCCA- } \\
\text { GATICCAATAAAATA }\end{array}$ & PUP14 DCR302 \\
\hline PUP74" & PUP14 in binary vector & PUP14 рСВ302 & Kan/Basta & amiR14_2R* $R$ & $\begin{array}{l}\text { GGTTGAAGAATCACGCTCGATATTIATIGG- } \\
\text { AATㄷGGGACAAATTG }\end{array}$ & PUP74 pСВ3О2 \\
\hline $35 S>A I C>P U I P 14$ & Ethanol-inducible binary & $O M 7-U C(5)$ & Kan/Kan & PUP14_LIC_f & $\begin{array}{l}\text { tagttggaatgggttcga ATCCATGGCTCAGAATCAA- } \\
\text { CAAC }\end{array}$ & Coln genomic \\
\hline OOOSALC $>$ F UT TI4 & vector (3) & Divi -LTC (J) & Rali/nani & PUP14_LIC_r & $\begin{array}{l}\text { ttatggagttgggttcgaa ATAAGCCATACGATTGTCTT- } \\
\text { TGTG }\end{array}$ & corv gerromic \\
\hline $35 S>A L C>P U P 14-G F P$ & Ethanol-inducible binary & $D M 7-1 / C(5)$ & Kan/Kan & PUP14_LIC_f & $\begin{array}{l}\text { tagttggaatgggttcga ATCCATGGCTCAGAATCAA- } \\
\text { CAAC }\end{array}$ & PUP14::PUP14 \\
\hline OSOSALCSTUT IU-GTH & vector $(3)$ & & hanindin & GFP_LIC_r & tattggagttgggttcga TTACTTGTACAGCTCGTCCATGC & $G F P$ \\
\hline 35S..PIUP1 & Binary vector for & nPIV?6 (36) & Kan/Kan & PUP1_LIC_F & tagttggaatgggttcgaa ACAGCAAGCAGCAAGAAGAA & Coln genomic \\
\hline $000 . .101+1$ & microsomes & $\operatorname{sr} L v<0(00)$ & Rairitian & PUP1_LIC_R & $\begin{array}{l}\text { ttatggagttgggttcgaa AGCAACATAATCACTAACAGG- } \\
\text { AAG }\end{array}$ & Corv gentiviní \\
\hline & & $\begin{array}{l}h b t:: L I C-H A \\
\text { (Genbank }\end{array}$ & & CXX2_LIC_f & $\begin{array}{l}\text { tagttggaatgggttcgaaTAAACAAATGGCTAATCTT- } \\
\text { CGTT }\end{array}$ & \\
\hline $\begin{array}{l}h b t:: C K X 2-H A ; \\
h b t:: C K X 2-G F P\end{array}$ & $\begin{array}{l}\text { Protoplast expression } \\
\text { vector }\end{array}$ & $\begin{array}{l}\text { KX510274), } \\
\text { hbt::LIC-GFP } \\
\text { (Genbank } \\
\text { KX510273) }\end{array}$ & Amp & CXX2_LIC_r & $\begin{array}{l}\text { ttatggagttgggttcgaaGATGTCTTGCCCTGGAGATAA- } \\
\text { CA }\end{array}$ & Col0 genomic \\
\hline & & $\begin{array}{l}\text { hbt::LIC-HA } \\
\text { (Genbank }\end{array}$ & & CKX2 $\Delta S P \_L I C \_f$ & $\begin{array}{l}\text { tagttggaatgggttcgaATGATTAAAATTGATT- } \\
\text { TACCTAAATCCC }\end{array}$ & \\
\hline 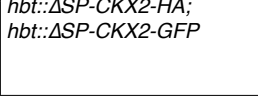 & $\begin{array}{l}\text { Protoplast expression } \\
\text { vector }\end{array}$ & $\begin{array}{l}\text { KX510274), } \\
\text { hbt::LIC-GFP } \\
\text { (Genbank } \\
\text { KX510273) }\end{array}$ & Amp & CXX2_LIC_r & $\begin{array}{l}\text { ttatggagttgggttcgaaGATGTCTTGCCCTGGAGATAA- } \\
\text { CA }\end{array}$ & Col0 genomic \\
\hline
\end{tabular}

\section{Table S2. Construct list.}

Lowercase font in primer sequence denote adaptor sequence, underlined nucleotides indicate mutations or insertions. 


\begin{tabular}{|c|c|c|c|c|c|c|}
\hline \multirow{2}{*}{ Name } & \multirow{2}{*}{ Purpose } & \multirow{2}{*}{ Parent vector } & \multirow{2}{*}{$\begin{array}{c}\text { Selection } \\
\text { (bacteria/plants) }\end{array}$} & \multicolumn{3}{|c|}{ Insert } \\
\hline & & & & Primer name & Sequence $5^{\prime}$ to $3^{\prime}$ & Template \\
\hline \multirow[b]{2}{*}{$\begin{array}{l}\text { hbt::CKX7-HA; } \\
\text { hbt::CKX7-GFP }\end{array}$} & \multirow[b]{2}{*}{$\begin{array}{l}\text { Protoplast expression } \\
\text { vector }\end{array}$} & \multirow{2}{*}{$\begin{array}{c}\text { hbt::LIC-HA } \\
\text { (Genbank } \\
\text { KX510274); } \\
\text { hbt::LIC-GFP } \\
\text { (Genbank } \\
\text { KX510273) }\end{array}$} & \multirow[b]{2}{*}{ Amp } & CXX7_LIC_f & tagttggaatgggttcgaaCACACACACCAAAATGATAGCT & \multirow[b]{2}{*}{ Col0 genomic } \\
\hline & & & & CXX7_LIC_r & $\begin{array}{l}\text { ttatggagttgggttcgaaAAGAGACCTATTGAAAATCTTT- } \\
\text { TGACC }\end{array}$ & \\
\hline \multirow{2}{*}{$h b t:: C K X 2-G F P-G P I$} & \multirow{2}{*}{$\begin{array}{l}\text { Protoplast expression } \\
\text { vector }\end{array}$} & \multirow{2}{*}{$\begin{array}{l}\text { hbt::LIC-GFP- } \\
\text { GPI (Genbank } \\
\text { KX510275) }\end{array}$} & \multirow{2}{*}{ Amp } & CXX2_LIC_f & $\begin{array}{l}\text { tagttggaatgggttcgaaTAAACAAATGGCTAATCTT- } \\
\text { CGTT }\end{array}$ & \multirow{2}{*}{ Col0 genomic } \\
\hline & & & & CXX2_LIC_r & $\begin{array}{l}\text { ttatggagttgggttcgaaGATGTCTTGCCCTGGAGATAA- } \\
\text { CA }\end{array}$ & \\
\hline \multirow{2}{*}{$h b t:: C K X 2-G P I$} & \multirow{2}{*}{$\begin{array}{l}\text { Protoplast expression } \\
\text { vector }\end{array}$} & \multirow{2}{*}{$h b t:: C K X 2-H A$} & \multirow{2}{*}{ Amp } & GPI_Pst_f & $\begin{array}{l}\text { AACGGTGGTTCCCGGTCACAATTCTCATTCGTCG- } \\
\text { CCGCCGTGCTCCTCCCTCTTCTTGTCTTTTTCTTC } \\
\text { TTCTCTGCCTAActgca }\end{array}$ & \multirow{2}{*}{$\begin{array}{c}\text { Stul Pstl } \\
\text { digested } \\
h b t:: C K X 2-H A\end{array}$} \\
\hline & & & & GPI_Pst_r & $\begin{array}{l}\text { gTTAGGCAGAGAAGAAGAAAAAGACAAGAA- } \\
\text { GAGGGAGGAGCACGGCGGCGACGAATGAG- } \\
\text { AATTGTGACCGGGAACCACCGTT }\end{array}$ & \\
\hline
\end{tabular}

\section{Table S2 (continued).}

Construct list. Lowercase font in primer sequence denote adaptor sequence, underlined nucleotides indicate mutations or insertions. 